Homology, Homotopy and Applications, vol.12(1), 2010, pp.367-400

\title{
LA COHOMOLOGIE TOTALE EST UN FONCTEUR DÉRIVÉ
}

\author{
FRANÇOIS LESCURE
}

(communicated by Johannes Huebschmann)

\begin{abstract}
We use a certain sheaf of associative rings to define a global Ext functor. We prove that the "cohomologie totale" which we defined in an earlier paper in an analytic way is given by this global Ext. We use this functorial definition to prove some results conjectured in earlier papers. We introduce the "anchor spectral sequence" and use it to give a precise description of the total cohomology for the special case of complex homogeneous spaces.
\end{abstract}

\section{Introduction, rappels et définitions.}

\subsection{Action holomorphe locale.}

Soit $M$ une variété analytique complexe et $G$ un groupe de Lie complexe d'élément neutre $e$; on rappelle qu'une $G$-action holomorphe locale sur $M$ est la double donnée d'un ouvert connexe $\Omega \subset G \times M$ qui soit un voisinage de $\{e\} \times M$ et d'une application holomorphe A: $\Omega \rightarrow M$ que :

a) Pour tout $\boldsymbol{m} \in M$, on a : $\mathrm{A}(e, \boldsymbol{m})=\boldsymbol{m}$.

b) Si $g$ et $g^{\prime}$ sont dans $G$ et que $\boldsymbol{m}$ appartient à $M$, alors dès que $\left(g^{\prime}, \boldsymbol{m}\right),\left(g g^{\prime}, \boldsymbol{m}\right)$ et $\left(g, \mathrm{~A}\left(g^{\prime}, \boldsymbol{m}\right)\right)$ sont tous trois dans $\Omega$ on a : $\mathrm{A}\left(g, \mathrm{~A}\left(g^{\prime}, \boldsymbol{m}\right)\right)=\mathrm{A}\left(g g^{\prime}, \boldsymbol{m}\right)$. Par raccourci, on dira que A est l'action locale. Lorsque $\Omega$ est $G \times M$ tout entier, on retombe sur le cas d'une $G$-action holomorphe de $G$ sur $M$. On dira que l'on a alors une véritable action. Utilisant les notations en usage dans ce dernier contexte, nous écrirons souvent $g \boldsymbol{m}$ au lieu de $\mathrm{A}(g, \boldsymbol{m})$.

Désignons maintenant par $T M$ le fibré tangent réel de $M$ naturellement holomorphe pour l' opérateur de multiplication complexe J qui provient de la structure analytique, et par $\mathfrak{g}_{0}$ l' algèbre de Lie du groupe $G$ qui est naturellement une algèbre de Lie complexe pour un opérateur de multiplication complexe J. Définissons alors, si $\mathbf{u} \in \mathfrak{g}_{0}$, un champ $\mathbf{u}_{M}$ sur $M$ par la formule :

$$
\forall \boldsymbol{m} \in M, \mathbf{u}_{M}(\boldsymbol{m})=\left[\frac{d}{d t} \exp (-t \mathbf{u}) \boldsymbol{m}\right]_{\mid t=0} \in \boldsymbol{T}_{\boldsymbol{m}}(M) .
$$

Dans ce qui suit on opposera la transformation infinitésimale holomorphe, i.e. la

L'auteur tient à remercier J. Huebschmann pour le temps qu'il lui a accordé. Received December 1, 2007, revised March 26, 2009; published on June 1, 2010. 2000 Mathematics Subject Classification: 32M05, 32M25, $17 \mathrm{~B} 66$.

Key words and phrases: Lie algebra of vector fields, complex vector fields, complex Lie groups, groups of automorphisms acting on complex spaces.

This article is available at http://intlpress.com/HHA/v12/n1/a19

Copyright (C) 2010, International Press. Permission to copy for private use granted. 
section holomorphe $\mathbf{u}_{M} \in H^{0}(M, T M)$ dans le fibré tangent réel holomorphe $T M$, et sa $(1,0)$-composante

$$
\mathbf{u}_{M}^{1,0}=\frac{1}{2}\left(\mathbf{u}_{M}-i \mathrm{~J} \mathbf{u}_{M}\right) \in H^{0}\left(M, T^{1,0} M\right) ;
$$

étant par ailleurs rappelé que : $(\mathrm{Ju})_{M}=\mathrm{J} \mathbf{u}_{M}$.

De manière plus générale, si $\mathbf{u} \in \mathfrak{g}_{\mathbb{C}} \stackrel{\text { def }}{=} \mathfrak{g}_{0} \otimes_{\mathbb{R}} \mathbb{C}$, on comprend le sens de $\mathbf{u}_{M} \in H^{0}\left(M, T M \otimes_{\mathbb{R}} \mathbb{C}\right)$. Enfin on prolonge par $\mathbb{C}$-linéarité l'opérateur $\mathrm{J}$ à $\mathfrak{g}_{\mathbb{C}}$ qui donne la décomposition en espaces propres $\mathfrak{g}_{\mathbb{C}}=\mathfrak{g} \oplus \overline{\mathfrak{g}}$, associée aux valeurs propres $+i$ et $-i$. La flèche $\mathfrak{g}_{0} \ni \mathbf{u} \mapsto \mathbf{u}_{M}$ est, [4], le morphisme d'ancrage qui vérifie $[\mathbf{u}, \mathbf{v}]_{M}=$ $\left[\mathbf{u}_{M}, \mathbf{v}_{M}\right]$, où ce dernier crochet est évidemment celui des champs de vecteurs tangents réels. On prolonge par $\mathbb{C}$-linéarité ce morphisme d'ancrage; mais arrivé ici on observe :

1er) Par la dérivation de Lie $\mathcal{L}_{\mathbf{u}_{M}}$, la représentation linéaire de $\mathfrak{g}_{0}$ dans les espaces de fonctions indéfiniment différentiables à valeurs complexes au dessus d'un ouvert $U \subset M$ n'est pas une représentation d'algèbre de Lie complexe $\mathfrak{g}_{0}$, i.e. encore, si $\varphi$ est une telle fonction locale : $\mathcal{L}_{\mathbf{J}_{\mathbf{u}}} \varphi \neq i \mathcal{L}_{\mathbf{u}_{M}} \varphi$. Toutefois la sous-représentation au sousespace des fonctions qui sont holomorphes est bien une représentation de l'algèbre de Lie complexe $\mathfrak{g}_{0}$.

2eme) Mais si on considère la décomposition $\mathfrak{g}_{0} \ni \mathbf{u}_{0}=\mathbf{u}^{1,0}+\mathbf{u}^{0,1}$, la projection $\mathfrak{g}_{0} \ni \mathbf{u}_{0} \mapsto \mathbf{u}^{1,0} \in \mathfrak{g}$ est un isomorphisme d'algèbre de Lie complexes. Observons que dans une carte locale holomorphe la $(1,0)$-partie $\mathbf{u}_{M}^{1,0}=\left(\mathbf{u}^{1,0}\right)_{M}$ de $\mathbf{u}_{M}$ qui n'est plus un champ réel s'écrit :

$$
\mathbf{u}_{M}^{1,0}=\sum f^{\alpha} \frac{\partial}{\partial z^{\alpha}}
$$

avec des $f^{\alpha}$ holomorphes. C'est un tel champ de $(1,0)$-vecteurs que l'on appellera champ holomorphe par opposition au champ réel :

$$
\mathbf{u}_{M}=\sum f^{\alpha} \frac{\partial}{\partial z^{\alpha}}+\sum \bar{f}^{\alpha} \frac{\partial}{\partial \bar{z}^{\alpha}}
$$

qu'on appellera transformation infinitésimale holomorphe, transformation infinitésimale $\mathbf{u}_{M}$ souvent confondue avec sa $(1,0)$-composante, i.e. avec le champ holomorphe $\mathbf{u}_{M}^{1,0}$ (ainsi l'écriture $\exp \left(t \mathbf{u}_{M}^{1,0}\right)$ pour les automorphismes locaux). Il est vrai que $\mathcal{L}_{\mathbf{u}_{M}}$ et $\mathcal{L}_{\mathbf{u}_{M}^{1,0}}$ agissent de la même manière sur les fonctions holomorphes (et même sur les $(p, 0)$ formes holomorphes en vertu de l'identité de Carrel et Liebermann). C'est précisément l'observation que les deux dérivés de Lie qui précèdent agissent différemment sur les fonctions indéfiniment différentiables qui fait l'intérêt de la différentielle $D$ de [19] ( $c f$. plus bas définition/Rappels 3), alors que la représentation du groupe de Lie complexe $G$ dans l'espace $\mathcal{C}^{\infty}(M, \mathbb{C})$ n'est pas holomorphe.

3eme) Mais, si $\mathbf{u} \in \mathfrak{g} \stackrel{\text { def }}{=} \mathfrak{g}^{1,0} \subset \mathfrak{g}_{\mathbb{C}}$, on voit que, via la dérivation de Lie $\varphi \longmapsto \mathcal{L}_{\mathbf{u}_{M}} \varphi$, le long du champ holomorphe $\mathbf{u}_{M}$, on définit bien une représentation de l'algèbre de Lie complexe $\mathfrak{g}$ dans le faisceau $\mathcal{C}^{\infty}(M, \mathbb{C})$ des germes de fonctions indéfiniment différentiables. Une chose essentielle est donc qu'ici et dans la suite, le morphisme d'ancrage n'est pas à valeurs dans les champs réels.

Mais alors, on se trouve précisément dans la situation d'un faisceau d'algèbres croisées (qui est presque celle donnée dans [4] d'une algébroïde, où est supposée, en 
sus, l'hypothèse d'être de type fini sur $\left.\mathcal{O}_{M}\right)$ en définissant $\operatorname{sur} \mathcal{C}_{M}^{\infty} \otimes_{\mathbb{C}} \mathfrak{g}$, une structure de faisceau d'algèbres de Lie au moyen ( $\varphi$ et $\psi$ étant des fonctions $\mathcal{C}^{\infty}$ locales) du crochet :

$$
\left[\varphi \otimes_{\mathbb{C}} \mathbf{u}, \psi \otimes_{\mathbb{C}} \mathbf{v}\right]=\left(\varphi \mathcal{L}_{\mathbf{u}_{M}} \psi\right) \otimes_{\mathbb{C}} \mathbf{v}-\left(\psi \mathcal{L}_{\mathbf{v}_{M}} \varphi\right) \otimes_{\mathbb{C}} \mathbf{u}+(\varphi \psi) \otimes_{\mathbb{C}}[\mathbf{u}, \mathbf{v}]
$$

On introduit aussi la sous-algèbre de Lie faisceautique $\boldsymbol{E}_{1}\left(\mathfrak{g}, \mathcal{O}_{M}\right)$ des germes de sections $\varphi \otimes_{\mathbb{C}} \mathbf{u}$ comme ci-dessus, mais où $\varphi$ est holomorphe. L'algèbre de Lie $\boldsymbol{E}_{1}\left(\mathfrak{g}, \mathcal{O}_{M}\right)$ étant alors localement libre de rang fini sur $\mathcal{O}_{M}$, on est exactement dans le cas d'une algébroïde complexe ([4] définition 2.1.2. et plus précisément l'exemple 4 de la page 372 ). Alors (toujours $[4])$ si $U(\mathfrak{g})=U(\mathfrak{g}, \mathbb{C})$ est l'algèbre enveloppante de l'algèbre de Lie complexe $\mathfrak{g}$, on définit le faisceau de $\mathbb{C}$-algèbres associatives $\boldsymbol{U}\left(\mathfrak{g}, \mathcal{O}_{M}\right)$ en posant : $\boldsymbol{U}\left(\mathfrak{g}, \mathcal{O}_{M}\right) \stackrel{\text { def }}{=} \mathcal{O}_{M} \otimes_{\mathbb{C}} U(\mathfrak{g}, \mathbb{C})$, où la structure annelée sur ce produit tensoriel est définie (par récurrence sur la "longueur" de D) par les formules de commutation :

$$
\begin{gathered}
\left(\varphi \otimes_{\mathbb{C}} \mathbf{D u}\right)\left(\psi \otimes_{\mathbb{C}} \mathbf{D}^{\prime}\right)=\left(\varphi \otimes_{\mathbb{C}} \mathbf{D}\right)\left(\mathcal{L}_{\mathbf{u}_{M}} \psi \otimes_{\mathbb{C}} \mathbf{D}^{\prime}\right)+\left(\varphi \otimes_{\mathbb{C}} \mathbf{D}\right)\left(\psi \otimes_{\mathbb{C}} \mathbf{u D}^{\prime}\right) \\
\left(\varphi \otimes_{\mathbb{C}} 1\right)\left(\psi \otimes_{\mathbb{C}} \mathbf{D}^{\prime}\right)=(\varphi \psi) \otimes_{\mathbb{C}} \mathbf{D}^{\prime}
\end{gathered}
$$

Ainsi on voit que, ce que nous désignons ici $\boldsymbol{U}\left(\mathfrak{g}, \mathcal{O}_{M}\right)$, est exactement ce qui serait désigné $\mathcal{D}\left(\mathbf{E}_{1}\left(\mathfrak{g}, \mathcal{O}_{M}\right)\right.$ ) dans $[4]$. Au moyen de $\varphi \mapsto \varphi \otimes_{\mathbb{C}} 1$ (resp. $\mathbf{D} \mapsto 1 \otimes_{\mathbb{C}} \mathbf{D}$ ), on identifie $\mathcal{O}_{M}$ (resp. le faisceau "constant" $U(\mathfrak{g}, \mathbb{C})$ ) à un sous-faisceau d'anneaux de $\boldsymbol{U}\left(\mathfrak{g}, \mathcal{O}_{M}\right)$. On écrit alors plus simplement $\varphi \mathbf{D}$ au lieu de $\varphi \otimes_{\mathbb{C}} \mathbf{D}$. La formule 3 affirme alors en substance que $\mathbf{u} \varphi=\mathcal{L}_{\mathbf{u}_{M}} \varphi+\varphi \mathbf{u}$, i.e. mutatis mutandis la classique formule de commutation " $X \varphi=\mathcal{L}_{X} \varphi+\varphi X$ " qui définit la structure annelée de $\mathcal{D}_{M}$.

Avec ces définitions, un $\boldsymbol{U}\left(\mathfrak{g}, \mathcal{O}_{M}\right)$-module à gauche n'est donc rien d'autre qu'un faisceau $\mathcal{F}$ de $\mathcal{O}_{M}$-module (non nécessairement cohérent ni même de type fini) avec un morphisme d'algèbre de Lie complexe $\gamma_{*}: \mathfrak{g} \rightarrow \operatorname{End}_{\mathbb{C}}(\mathcal{F})$ qui vérifie $(\sigma$ section locale de $\mathcal{F}$ ) la relation de commutation :

$$
\gamma_{*}(\mathbf{u})(\varphi \sigma)=\mathcal{L}_{\mathbf{u}_{M}} \varphi \sigma+\varphi \gamma_{*}(\mathbf{u}) \sigma
$$

Dans la suite, le contexte étant clair, on notera aussi plus brièvement $\gamma_{*}(\mathbf{u}) \boldsymbol{s}$ par $\mathbf{u} \cdot \boldsymbol{s}$. Il est bon, ici, de remarquer qu'alors que $\mathbb{C}$ est dans le centre de $U(\mathfrak{g}, \mathbb{C})$, le sous-faisceau

$$
\mathcal{O}_{M}=\mathcal{O}_{M} \otimes_{\mathbb{C}} \mathbb{C} \subset \boldsymbol{U}\left(\mathfrak{g}, \mathcal{O}_{M}\right)
$$

n'est évidemment pas, en général, dans le centre de $\boldsymbol{U}\left(\mathfrak{g}, \mathcal{O}_{M}\right)$. Enfin précisons ici, dès maintenant, une notation constamment utilisée dans la suite : si $\mathcal{F}$ est un $\boldsymbol{U}\left(\mathfrak{g}, \mathcal{O}_{M}\right)$ module, $\mathcal{F}^{\mathfrak{g}}$ en désignera le sous-faisceau des germes de sections $\mathfrak{g}$-invariantes, i.e. des germes de sections $\sigma$ tels que $\gamma_{*}(\mathbf{u}) \sigma=0$ pour tous les $\mathbf{u} \in \mathfrak{g}$.

\subsection{Choses vues à gauche et à droite.}

On peut analogiquement définir des $\boldsymbol{U}\left(\mathfrak{g}, \mathcal{O}_{M}\right)$-modules à droite, au sens de la définition classique et générale d'un faisceau de modules à droite sur un faisceau d'anneaux. Plus concrétement, on voit aisément qu'un $\boldsymbol{U}\left(\mathfrak{g}, \mathcal{O}_{M}\right)$-modules à droite n'est rien d'autre qu'un faisceaux $\mathcal{F}$ de $\mathcal{O}_{M}$-modules à gauche (et donc en fait à droite puisque $\mathcal{O}_{M}$ est un faisceau d'anneaux commutatifs; la multiplication s'écrira 
donc en multipliant à droite par les scalaires) avec une $\mathfrak{g}$-action à droite, que nous contenterons de définir sur les germes de $\mathcal{F}$, qui vérifie :

$$
\begin{aligned}
\sigma \cdot[\mathbf{u}, \mathbf{v}] & =(\sigma \cdot \mathbf{u}) \cdot \mathbf{v}-(\sigma \cdot \mathbf{v}) \cdot \mathbf{u} \\
(\sigma \cdot \mathbf{u}) \cdot f & =\sigma \cdot\left(\mathcal{L}_{\mathbf{u}_{M}} f\right)+(\sigma \cdot f) \cdot \mathbf{u}
\end{aligned}
$$

N.B. En fait, et contrairement au cas des $\mathcal{D}_{M}$-modules, le fait d'être un $\boldsymbol{U}\left(\mathfrak{g}, \mathcal{O}_{M}\right)$ module à droite est équivalent au fait d'être aussi un $\boldsymbol{U}\left(\mathfrak{g}, \mathcal{O}_{M}\right)$-module à gauche.

En effet soit $\mathcal{F}$ un $\boldsymbol{U}\left(\mathfrak{g}, \mathcal{O}_{M}\right)$-module à gauche. Si $f$ est dans $\mathcal{O}_{M}$ posons : $\sigma \cdot f=f \sigma$ et $\sigma \cdot \mathbf{u}=-\mathbf{u} \cdot \sigma$. Alors on a :

$$
(\sigma \cdot \mathbf{u}) \cdot f=-f(\mathbf{u} \sigma)=-\mathbf{u}(f \sigma)+\left(\mathcal{L}_{\mathbf{u}_{M}} f\right) \sigma=\sigma \cdot\left(\mathcal{L}_{\mathbf{u}_{M}} f\right)+(\sigma \cdot f) \cdot \mathbf{u} .
$$

On voit, alors, par quel procédé réciproque du précédent, définir un $\boldsymbol{U}\left(\mathfrak{g}, \mathcal{O}_{M}\right)$ module à gauche à partir d'un $\boldsymbol{U}\left(\mathfrak{g}, \mathcal{O}_{M}\right)$-module à droite. De ces deux structures, l'une à gauche, l'autre à droite, on dira qu'elles sont miroirs l'une de l'autre.

Cette distinction radicale entre $\boldsymbol{U}\left(\mathfrak{g}, \mathcal{O}_{M}\right)$-modules et $\mathcal{D}_{M}$-modules tient à ce que, si $\mathcal{D}_{M}$ n'est en général pas isomorphe à $\mathcal{D}_{M}^{o p p}$, on peut vérifier, au moyen de la formule :

$$
\left(f \mathbf{u}_{1} \cdots \mathbf{u}_{p}\right)^{\top}=(-1)^{p} \mathbf{u}_{p} \cdots \mathbf{u}_{1} f
$$

que la transformation $\bullet^{\top}$ définit un isomorphisme de $\boldsymbol{U}\left(\mathfrak{g}, \mathcal{O}_{M}\right)^{\text {opp }} \operatorname{sur} \boldsymbol{U}\left(\mathfrak{g}, \mathcal{O}_{M}\right)$. Si $\mathcal{F}$ est un $\boldsymbol{U}\left(\mathfrak{g}, \mathcal{O}_{M}\right)$-module à gauche, son action miroir à droite est donc définie par $\sigma \cdot \boldsymbol{D}=\boldsymbol{D}^{\top} \cdot \sigma$. Ceci dit, il y a quand même une équivalence de catégories entre les $\mathcal{D}_{M}$-modules à gauche et les $\mathcal{D}_{M}$-modules à droite qui fait intervenir la structure de $\mathcal{D}_{M}$-module à droite du faisceau des formes différentielles de degré maximal. Et qui ne provient pas, en général, d'un anti-isomorphisme d'algèbre de $\mathcal{D}_{M}$.

\subsection{Objet du présent travail.}

On ne rappelle pas ici ( $c f . \quad[\mathbf{1 9}]$ et [16]), ni la définition d'un fibré holomorphe $G$-équivariant, et, a fortiori ni non plus, celle ( $c f$. [19] note 8 en bas de la page 112) d'un faisceau analytique cohérent localement $G$-équivariant, tous objets pour lesquels il est possible de définir la cohomologie totale. Elle sera définie plus bas ( cf. Définition/Rappels 3) comme dans [19], au moyen des "cochaînes totales", objets qui s'apparentent aux formes différentielles usuelles.

Leur grand intérêt est surtout en fait de pouvoir définir un analogue de la dérivée de Lie $\mathcal{L}_{X}$ et de la différentielle de de Rham pour lesquelles on ait (cf. [19] formule (19) page 112) un analogue de la formule $\mathcal{L}_{X}=i(X) d+d i(X)$.

On a pu alors observer que la suite de ces foncteurs de cohomologie totale constituait un objet connu des algébristes : en l'occurrence une suite connectée ([12]) de foncteurs additifs, le zéroième étant même exact à gauche. C'est une condition nécessaire (mais non suffisante) pour que ce soit celle des foncteurs dérivés du zéroième. D'où la question naturelle, posée dans [19] (toujours dans la note 8 du bas de la page 112) de savoir si cette cohomologie totale était un foncteur dérivé. A cette fin, ayant alors précisé la notion plus générale de $\mathcal{O}_{M}$-module $\mathfrak{g}$-équivariant pour l'action infinitésimale d'une algèbre de Lie complexe $\mathfrak{g}$, cette question nous amène naturellement à considérer leur catégorie, avec des morphismes évidents, qui s'avère, en fait, n'être rien d'autre que celle des $\boldsymbol{U}\left(\mathfrak{g}, \mathcal{O}_{M}\right)$-modules déjà définie et étudiée dans $[\mathbf{4}]$ 
(voir aussi [15] dans un contexte "dédoublé"). Ce simple raccordement de la cohomologie totale avec la théorie des algébroïdes de [4] permet d'interpréter la cohomologie totale comme un "Ext global". Ainsi, (théorème 3.3) si $E$ est un fibré holomorphe $G$-équivariant la cohomologie totale $\mathbf{H}^{\bullet}(\mathfrak{g}, E)$ ( $c f$. Proposition/Rappels 33.2 ) vaut

$$
\operatorname{Ext}_{\boldsymbol{U}\left(\mathfrak{g}, \mathcal{O}_{M}\right)}\left(M ; \mathcal{O}_{M}, E\right) .
$$

Cet article qui est donc une "algébrisation" de l'article [19], donne aussi des indications brèves (par des procédés tout à fait standards et classiques) pour démontrer (cf. 3.8 et 3.9) des résultats indiqués seulement comme plausibles dans [19] (note 20 bas de page 129). On approche aussi la cohomologie totale au moyen d'une suite spectrale qu'on illustre concrétement dans le cas homogène. On évoque aussi les analogies et dissemblances avec les $\mathcal{D}$-modules et on évoque des résolutions "quasi-projectives" pour une classe naturelle de $\boldsymbol{U}\left(\mathfrak{g}, \mathcal{O}_{M}\right)$-modules.

Enfin et pour finir, il semble que beaucoup de notions et d'objets ne soient définissables qu'au moyen de l'algèbre homologique relative. On ne donne ici (pour contenir le volume de l'article) que quelques éléments qui suggèrent déjà l'intérêt de prospecter dans cette direction. On espère surtout que cette première présentation algébrisée de notions d'abord définies analytiquement permettra à de véritables algébristes de s'intéresser à la cohomologie totale et d'y apporter éventuellement leur contribution.

\section{Premières remarques sur les $U\left(\mathfrak{g}, \mathcal{O}_{M}\right)$-modules ; "géométri- cité" de certains exemples.}

\subsection{Premiers exemples naturels.}

Le premier exemple - important pour la suite - de $\boldsymbol{U}\left(\mathfrak{g}, \mathcal{O}_{M}\right)$-module à gauche qui vient à notre rencontre est le faisceau structural $\mathcal{O}_{M}$ avec " $\varphi \cdot f=\varphi f$ " et $\boldsymbol{\gamma}_{*}(\mathbf{u}) f \stackrel{\text { def }}{=}$ $\mathcal{L}_{\mathbf{u}_{M}} f$. Après celui-ci, l'exemple le plus simple et qui en est la généralisation la plus naturelle est donnée par la :

Définition 2.1. Action issue de la base. Soit $\boldsymbol{L}$ un faisceau sur $\mathbb{C}$. Alors sur le $\mathcal{O}_{M-}$ module à gauche $\mathcal{O}_{M} \otimes_{\mathbb{C}} \boldsymbol{L}$, on définit une structure de $\boldsymbol{U}\left(\mathfrak{g}, \mathcal{O}_{M}\right)$-module à gauche au moyen, si $\boldsymbol{a}$ est une section locale de $\boldsymbol{L}$, de la formule:

$$
\gamma_{*}(\mathbf{u})\left(\varphi \otimes_{\mathbb{C}} \boldsymbol{a}\right) \stackrel{\text { def }}{=} \mathcal{L}_{\mathbf{u}_{M}} \varphi \otimes_{\mathbb{C}} \boldsymbol{a} .
$$

On dira que cette action sur $\mathcal{O}_{M} \otimes_{\mathbb{C}} \boldsymbol{L}$ est "l'action issue de la base".

Philosophiquement, on pense prioritairement au cas où $\boldsymbol{L}$ est un système local et où, donc, toutes les sections "philosophiquement localement constantes" sont "g-invariantes", i.e. telles que $\boldsymbol{\gamma}_{*}(\mathbf{u}) \boldsymbol{a}=0$, lorsque $\mathcal{O}_{M} \otimes_{\mathbb{C}} \boldsymbol{L}$ est le fibré holomorphe sous-jacent au système local complexe $\boldsymbol{L}$. L'operateur $\boldsymbol{\gamma}_{*}(\mathbf{u})$ est la dérivée covariante le long de $\mathbf{u}_{M}$ pour la connexion holomorphe intégrable définie classiquement par ces données. Signalons que cette philosophie a ses limites (potentiels de multicouches...).

On définira plus bas, de manière plus générale, la classe des $\boldsymbol{U}\left(\mathfrak{g}, \mathcal{O}_{M}\right)$-modules avec action issue de la base de telle manière, évidemment, que le $\mathcal{O}_{M}$-module à gauche $\mathcal{O}_{M} \otimes_{\mathbb{C}} \boldsymbol{L}$ défini immédiatement plus haut, soit dans cette dîte classe plus générale. On généralise la définition 2.1 dans une autre direction en considérant le faisceau, 
désigné $U\left(\mathfrak{g}, \mathbb{C}_{M}\right)$, de $\mathbb{C}$-algèbres des germes d'applications localement constantes de $M$ à valeurs dans $U(\mathfrak{g}, \mathbb{C})$. Supposons alors que $\boldsymbol{L}$ soit un $U\left(\mathfrak{g}, \mathbb{C}_{M}\right)$-module. (On pense "philosophiquement" à un système local " $G$ "-équivariant, c'est-à-dire tel, si $\boldsymbol{a}$ est une section, "philosophiquement localement constante" de $\boldsymbol{L}$ au dessus de $U$, qu'alors $\boldsymbol{\gamma}(g) \boldsymbol{a}$ soit encore une section "localement constante" au dessus de $g U$.) Le $\mathcal{O}_{M}$-module (à gauche) $\mathcal{O}_{M} \otimes_{\mathbb{C}} \boldsymbol{L}$ est alors naturellement un $\boldsymbol{U}\left(\mathfrak{g}, \mathcal{O}_{M}\right)$-module à gauche par "extension des scalaires" via l'isomorphisme canonique :

$$
\mathcal{O}_{M} \otimes_{\mathbb{C}} \boldsymbol{L} \approx \boldsymbol{U}\left(\mathfrak{g}, \mathcal{O}_{M}\right) \otimes_{U\left(\mathfrak{g}, \mathbb{C}_{M}\right)} \boldsymbol{L} .
$$

Dans un premier cas particulier où $\mathfrak{g}$ agit trivialement sur $\boldsymbol{L}$, i.e. $\boldsymbol{\gamma}_{*}(\mathbf{u}) \boldsymbol{a}=0$ pour toute section locale $\boldsymbol{a}$ de $\boldsymbol{L}$, on voit que l'on a fait rien d'autre que de retrouver l'action issue de la base de la définition 2.1 .

Un deuxième cas particulier apparaît naturellement lorsque l'on considère un espace vectoriel $\boldsymbol{V}$ qui est un $U\left(\mathfrak{g}, \mathbb{C}\right.$ )-module. Le faisceau constant $\mathbf{V}_{M}$ sur $M$ qui lui est associé est un $U\left(\mathfrak{g}, \mathbb{C}_{M}\right)$-module. En faisant la construction qui précède avec $\boldsymbol{L}=\mathbf{V}_{M}$ on voit que le $\mathcal{O}_{M}$-module globalement trivial : $\mathcal{O}_{M} \otimes_{\mathbb{C}} \mathbf{V}_{M}$ est encore un $\boldsymbol{U}\left(\mathfrak{g}, \mathcal{O}_{M}\right)$-module. On précise l'action de $\mathbf{u}$ par la formule :

$$
\gamma_{*}(\mathbf{u})[f \otimes \boldsymbol{v}]=\mathcal{L}_{\mathbf{u}_{M}} f \otimes \boldsymbol{v}+f \otimes \mathbf{u} \boldsymbol{v}
$$

ce $\boldsymbol{U}\left(\mathfrak{g}, \mathcal{O}_{M}\right)$-module s'interprète comme celui des fonctions holomorphes et localement à valeurs dans un sous-espace vectoriel de dimension finie de $\mathbf{V}$. Il va là encore de soi, que si la g-action sur $\mathbf{V}$ n'est pas intégrable en une représentation de $G$ dans $\mathbf{V}$, alors $\mathcal{O}_{M} \otimes_{\mathbb{C}} \mathbf{V}$ n'est pas nécessairement sous-jacent à un fibré holomorphe sur $M$ muni d'une $G$-action locale équivariante. L'intégrabilité est toutefois assurée si $\operatorname{dim}_{\mathbb{C}} \mathbf{V}<+\infty$.

Dans la suite il arrivera aussi que $\mathbb{C}$ désigne le système local complexe $\mathbb{C}_{M}$ de rang 1 des sections localement constantes à valeurs dans $\mathbb{C}$.

Nota Bene : Lorsqu'on a appelé $R$ la représentation de $\mathfrak{g}$ dans $\mathbf{V}$ qui en définit la structure de $U(\mathfrak{g}, \mathbb{C})$-module on préfèrera désigner le $U\left(\mathfrak{g}, \mathcal{O}_{M}\right)$-module

$$
\boldsymbol{U}\left(\mathfrak{g}, \mathcal{O}_{M}\right) \otimes_{U\left(\mathfrak{g}, \mathbb{C}_{M}\right)} \mathbf{V}_{M}
$$

par $\mathcal{O}_{M} \otimes_{\mathbb{C}} \mathbf{V}^{R}$, ou même encore, si le contexte est vraiment clair, par $\mathcal{O}_{M} \otimes_{\mathbb{C}} \mathbf{V}$. Enfin, on vérifie aisément :

(i) Le foncteur $\boldsymbol{U}\left(\mathfrak{g}, \mathcal{O}_{M}\right) \otimes_{U\left(\mathfrak{g}, \mathbb{C}_{M}\right)}$ - entre catégories de faisceaux de

$$
\operatorname{Mod}\left(U\left(\mathfrak{g}, \mathbb{C}_{M}\right)\right) \rightarrow \operatorname{Mod}\left(\boldsymbol{U}\left(\mathfrak{g}, \mathcal{O}_{M}\right)\right)
$$

est l'adjoint à gauche du foncteur "d'oubli" $\operatorname{Mod}\left(\boldsymbol{U}\left(\mathfrak{g}, \mathcal{O}_{M}\right)\right) \rightarrow \operatorname{Mod}\left(U\left(\mathfrak{g}, \mathbb{C}_{M}\right)\right)$.

(ii) On a une version "faisceautisée" de l'adjonction qui précède, i.e. un isomorphisme fonctoriel de faisceaux :

$$
\mathcal{H o m}_{\boldsymbol{U}\left(\mathfrak{g}, \mathcal{O}_{M}\right)}\left(\boldsymbol{U}\left(\mathfrak{g}, \mathcal{O}_{M}\right) \otimes_{U\left(\mathfrak{g}, \mathbb{C}_{M}\right)} \boldsymbol{L}, \mathcal{F}\right) \approx \mathcal{H} m_{U\left(\mathfrak{g}, \mathbb{C}_{M}\right)}(\boldsymbol{L}, \mathcal{F}) .
$$

(iii) Dès lors que $M$ a un nombre fini de composantes connexes, le foncteur

$$
V \mapsto \mathcal{O}_{M} \otimes_{\mathbb{C}} \mathbf{V}_{M}
$$

comme plus haut, de $\operatorname{Mod}(U(\mathfrak{g}, \mathbb{C})) \rightarrow \operatorname{Mod}\left(\boldsymbol{U}\left(\mathfrak{g}, \mathcal{O}_{M}\right)\right)$ est l'adjoint à gauche du foncteur "espace des sections globales" : $H^{0}(M,-)$ qui est à valeurs dans les espaces vectoriels qui sont des $\mathfrak{g}$-modules. 
Donnons enfin quelques exemples aussi simples qu'importants de cette situation :

(a) Par l'extension naturelle au produit extérieur de la représentation adjointe $\mathbf{V}=\wedge^{p} \mathfrak{g}$ devient un $U(\mathfrak{g}, \mathbb{C})$-module au moyen de la formule :

$$
\mathbf{u} \cdot\left(\mathbf{u}_{1} \wedge \cdots \wedge \mathbf{u}_{p}\right)=\sum \mathbf{u}_{1} \wedge \cdots \wedge \operatorname{ad} \mathbf{u}\left(\mathbf{u}_{k}\right) \wedge \cdots \wedge \mathbf{u}_{p}
$$

On posera alors $\boldsymbol{E}_{p}\left(\mathfrak{g}, \mathcal{O}_{M}\right) \stackrel{\text { def }}{=} \mathcal{O}_{M} \otimes_{\mathbb{C}} \wedge^{p} \mathfrak{g}$. Par multilinéarité la transformation $\mathbf{u} \mapsto \mathbf{u}_{M}$ se prolonge en un morphisme de $\boldsymbol{U}\left(\mathfrak{g}, \mathcal{O}_{M}\right)$-modules :

$$
\boldsymbol{E}_{p}\left(\mathfrak{g}, \mathcal{O}_{M}\right) \rightarrow \wedge^{p} \boldsymbol{T}^{1,0} M
$$

L'image, fort utile, de ce morphisme, sera appelée le $p^{\text {eme }}$ faisceau de Killing et sera désignée $\mathcal{K}^{p} \subset \wedge^{p} \boldsymbol{T}^{1,0}(M)$.

Pour $p=1$, on écrira plus brièvement $\mathcal{K} \stackrel{\text { def }}{=} \mathcal{K}^{1} \hookrightarrow T^{1,0} M$, et on l'appellera le faisceau de Killing. Observons qu'on peut aussi faire agir trivialement $\mathfrak{g}$ sur $\wedge^{p} \mathfrak{g}$; et donc, comme dans la définition 1 , faire de $\boldsymbol{E}_{p}\left(\mathfrak{g}, \mathcal{O}_{M}\right)$ un $\boldsymbol{U}\left(\mathfrak{g}, \mathcal{O}_{M}\right)$-module avec une action "issue de la base" à savoir naturellement associée au système local globalement constant $\wedge^{p} \mathfrak{g}$.

Le contexte permettra de savoir laquelle de ces deux structures de $\boldsymbol{U}\left(\mathfrak{g}, \mathcal{O}_{M}\right)$ modules on considère. Précisons toutefois, pour la suite, la première lorsque $p=n=$ $\operatorname{dim}_{\mathbb{C}} \mathfrak{g}$. Pour cela considérons la forme "module" $\triangle \in \mathfrak{g}^{*}$ définie par : $\triangle \mathbf{u}=\operatorname{tr}[\operatorname{adu}]$. Si $V_{n} \neq 0$ est dans $\wedge^{n} \mathfrak{g}$ et qu'alors $\varphi V_{n}$ est une section locale de $\boldsymbol{E}_{n}\left(\mathfrak{g}, \mathcal{O}_{M}\right)$, alors

$$
\gamma_{*}(\mathbf{u})\left(\varphi V_{n}\right)=\left(\mathbf{u}_{M} \cdot \varphi+\varphi \triangle \mathbf{u}\right) V_{n} .
$$

Autrement dit, $\boldsymbol{E}_{n}\left(\mathfrak{g}, \mathcal{O}_{M}\right)$ est le $\boldsymbol{U}\left(\mathfrak{g}, \mathcal{O}_{M}\right)$-module "tordu" $\mathcal{O}_{M}^{\triangle}$.

(b) Par l'extension naturelle de la représentation coadjointe, le produit extérieur

$$
C^{p}(\mathfrak{g}, \mathbb{C}) \stackrel{\text { def }}{=} \wedge^{p} \mathfrak{g}^{*}
$$

devient un $U(\mathfrak{g}, \mathbb{C})$-module par une formule mutatis mutandis comme plus haut en remplaçant ad par ăd : Le faisceau

$$
\boldsymbol{C}^{p}\left(\mathfrak{g}, \mathcal{O}_{M}\right) \stackrel{\text { def }}{=} \mathcal{O}_{M} \otimes_{\mathbb{C}} C^{p}(\mathfrak{g}, \mathbb{C})
$$

s'appelle traditionnellement faisceau des germes de p-cochaînes holomorphes à valeurs dans le faisceau structural et nous noterons ici

$$
\mathcal{E}^{p, q}\left(\mathfrak{g}, \mathcal{O}_{M}\right) \stackrel{\text { def }}{=} C^{p}(\mathfrak{g}, \mathbb{C}) \otimes_{\mathbb{C}} \mathcal{E}_{M}^{0, q}
$$

le faisceau des $(p, q)$-cochaînes totales à valeurs dans le faisceau structural, et

$$
\mathcal{E}^{n}\left(\mathfrak{g}, \mathcal{O}_{M}\right)=\oplus_{p+q=n} \mathcal{E}^{p, q}\left(\mathfrak{g}, \mathcal{O}_{M}\right)
$$

le faisceau des $n$-cochaînes totales qui, contrairement à ici, était précisément noté $\boldsymbol{C}^{n}\left(\mathfrak{g}, \mathcal{O}_{M}\right)$ dans $[\mathbf{1 6}],[\mathbf{1 9}]$, et $[\mathbf{2 1}]$.

\subsection{Exemples "non géométriques".}

La propriété d'être un $\boldsymbol{U}\left(\mathfrak{g}, \mathcal{O}_{M}\right)$-module n'est pas exactement identique au fait d'être un $\mathcal{O}_{M}$-module muni d'une action locale de $G$, comme on le montre ici par des exemples alors qualifiés de "non géométriques". C'est évidemment cette observation 
qui permet de mieux mesurer le déplacement de point de vue qui consiste à faire l'étude de la géométrie holomorphe équivariante via celle des $\boldsymbol{U}\left(\mathfrak{g}, \mathcal{O}_{M}\right)$-modules.

Afin, par un premier exemple, de préciser ce point, considérons un espace vectoriel réel $F_{0}$ de dimension $2 r$, et un opérateur "de multiplication complexe" J: $F_{0} \rightarrow F_{0}$ vérifiant $\mathrm{J}^{2}=-\mathrm{Id}$ qui définit un espace vectoriel complexe $F$ d'espace sous-jacent $F_{0}$ avec $\operatorname{dim}_{\mathbb{C}} F=r$.

On appellera espace conjugué de $F$, noté $\bar{F}$, le même espace sous-jacent $F_{0}$ mais avec la multiplication complexe $\mathrm{J}_{\bar{F}} \stackrel{\text { def }}{=}-\mathrm{J}$. On vérifie, si $u \in \operatorname{End}_{\mathbb{R}}\left(F_{0}\right)$ que

$$
u \in \operatorname{End}_{\mathbb{C}}(F) \Leftrightarrow u \in \operatorname{End}_{\mathbb{C}}(\bar{F})
$$

Mais, alors que la multiplication par $i$ de $u$ considéré comme élément de l'espace vectoriel complexe $\operatorname{End}_{\mathbb{C}}(F)$ est $\mathrm{J} \circ u$, celle de $i$ par $u$ considéré comme élément de l'espace vectoriel complexe $\operatorname{End}_{\mathbb{C}}(\bar{F})$, est $-\mathrm{J} \circ u$, si bien que, par restriction, la transformation

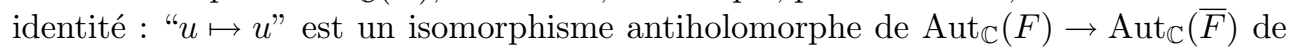
groupes de Lie complexes.

a) Fibrés antiholomorphes. Si, maintenant, $E \stackrel{\pi}{\rightarrow} M$ est un fibré vectoriel holomorphe de rang $r$, le même fibré vectoriel réel sous-jacent avec la structure complexe conjuguée sur chaque fibre vectorielle que l'on désignera $\bar{E}$ est donc définissable par des fonctions de transitions $g_{\alpha \beta}: U_{\alpha} \cap U_{\beta} \rightarrow \operatorname{Aut}_{\mathbb{C}}(\bar{F})$ antiholomorphes à valeurs dans $\operatorname{Aut}_{\mathbb{C}}(\bar{F})$.

Le faisceau $\mathcal{E}(\bar{E})$ des sections $\mathcal{C}^{\infty}$ dans $\bar{E}$ est naturellement un $\mathcal{E}_{M}$-module et, aussi par là même $\left(\mathcal{O}_{M} \subset \mathcal{E}_{M}\right)$, un $\mathcal{O}_{M}$-module. Par ailleurs, sans l'aide d'une connexion linéaire auxiliaire, il est possible de définir un opérateur $\overline{\mathcal{O}}_{M}$-linéaire

$$
\partial: \mathcal{E}_{M}(\bar{E}) \rightarrow \mathcal{E}_{M}^{1,0}(\bar{E})
$$

Si maintenant $\mathbf{u} \in \mathfrak{g}$ (et donc $\mathbf{u}_{M}$ un champ de (1,0)-vecteurs) en posant, pour tout germe $\sigma$ dans $\mathcal{E}_{M}(\bar{E})$,

$$
\left.\mathbf{u} \cdot \sigma \stackrel{\text { def }}{=} \mathbf{u}_{M}\right\rfloor \partial \sigma
$$

où $\left.\mathbf{u}_{M}\right\rfloor$ ( $c f$. annexe brève $\S 5$ ) est le produit intérieur par $\mathbf{u}_{M}$, on vérifie que l'on obtient bien un $\boldsymbol{U}\left(\mathfrak{g}, \mathcal{O}_{M}\right)$-module sur $\mathcal{E}_{M}(\bar{E})$. Il va de soi que l'existence de cette structure de $\boldsymbol{U}\left(\mathfrak{g}, \mathcal{O}_{M}\right)$-module ne préjuge en rien celle d'une $G$-équivariance infinitésimale "géométrique" (ou locale) sur $\bar{E}$. Autrement dit aussi, on n'a pas pour les fibrés véctoriels, même de rang fini, le pendant du résultat d'intégration de Palais, [24], pour les actions locales sur la base.

Toujours pour souligner cette opposition entre $\mathfrak{g}$-actions qui proviennent d'une "G-équivariance" et celles qui n'en proviennent pas, indiquons que l'on est naturellement amené à considérer sur $\boldsymbol{U}\left(\mathfrak{g}, \mathcal{O}_{M}\right)$ la structure $G$-équivariante suivante : Si $f \in H^{0}\left(U, \mathcal{O}_{M}\right)$ et si $f_{\mathbf{D}} \in H^{0}\left(U, \boldsymbol{U}\left(\mathfrak{g}, \mathcal{O}_{M}\right)\right)$, on définit $\gamma(g)[f \mathbf{D}]$ comme

$$
(\gamma(g) f)(\operatorname{Ad}(g) \mathbf{D}) \in H^{0}\left(g U, \boldsymbol{U}\left(\mathfrak{g}, \mathcal{O}_{M}\right)\right)
$$

Infinitésimalement cela revient à faire de $\boldsymbol{U}\left(\mathfrak{g}, \mathcal{O}_{M}\right)$ un module à sur lui même au moyen de

$$
\mathbf{u} \cdot(f \mathbf{D})=\mathcal{L}_{\mathbf{u}_{M}} f \mathbf{D}+f(\mathbf{u D}-\mathbf{D u})
$$


On conviendra de désigner par ${ }^{\text {ad }} \boldsymbol{U}\left(\mathfrak{g}, \mathcal{O}_{M}\right)$, voire même seulement ${ }^{\text {ad }} \boldsymbol{U}$, ce $\boldsymbol{U}\left(\mathfrak{g}, \mathcal{O}_{M}\right)$ module à gauche pour lequel l'inclusion $\mathcal{O}_{M} \hookrightarrow{ }^{\text {ad }} \boldsymbol{U}$ est $\boldsymbol{U}\left(\mathfrak{g}, \mathcal{O}_{M}\right)$-linéaire.

Si $p \geqslant 0$, on désigne par $\boldsymbol{U}_{p}\left(\mathfrak{g}, \mathcal{O}_{M}\right)$ le sous $\mathcal{O}_{M}$-module de $\boldsymbol{U}\left(\mathfrak{g}, \mathcal{O}_{M}\right)$ engendré par les $\mathbf{u}_{1} \cdots \mathbf{u}_{q}$ avec $q \leqslant p . \boldsymbol{U}_{p}\left(\mathfrak{g}, \mathcal{O}_{M}\right)$ est un $\boldsymbol{U}\left(\mathfrak{g}, \mathcal{O}_{M}\right)$-sous-module de ${ }^{\text {ad }} \boldsymbol{U}\left(\mathfrak{g}, \mathcal{O}_{M}\right)$ mais pas de ${ }^{g} \boldsymbol{U}\left(\mathfrak{g}, \mathcal{O}_{M}\right)$ défini dans ce qui suit immédiatement.

b) Le module ${ }^{g} \boldsymbol{U}$. En opposition avec cette $G$-action locale, on peut considérer sur $\boldsymbol{U}\left(\mathfrak{g}, \mathcal{O}_{M}\right)$ la structure $\boldsymbol{U}\left(\mathfrak{g}, \mathcal{O}_{M}\right)$-module à gauche sur lui même qui provient de la multiplication à gauche i.e. donnée par

$$
\mathbf{u} \cdot(f \mathbf{D})=\mathcal{L}_{\mathbf{u}_{M}} f \mathbf{D}+f \mathbf{u D} .
$$

On obtient ainsi, comme on peut s'en convaincre, un deuxième exemple de $\boldsymbol{U}\left(\mathfrak{g}, \mathcal{O}_{M}\right)$ module qui n'est point sous-jacent à une $G$-action holomorphe locale. On conviendra de désigner par ${ }^{g} \boldsymbol{U}\left(\mathfrak{g}, \mathcal{O}_{M}\right)$, ou même seulement ${ }^{g} \boldsymbol{U}$, ce $\boldsymbol{U}\left(\mathfrak{g}, \mathcal{O}_{M}\right)$-module à gauche pour lequel l'inclusion d'anneau $\mathcal{O}_{M} \hookrightarrow{ }^{g} \boldsymbol{U}$ n'est plus un $\boldsymbol{U}\left(\mathfrak{g}, \mathcal{O}_{M}\right)$-morphisme. Si on considère le module $\boldsymbol{U}^{d}\left(=\boldsymbol{U}\left(\mathfrak{g}, \mathcal{O}_{M}\right)\right)$ à droite défini par la multiplication à droite de $\boldsymbol{U}\left(\mathfrak{g}, \mathcal{O}_{M}\right)$, on prendra garde que ce n'est point le miroir de ${ }^{g} \boldsymbol{U}$. Le miroir à droite de ${ }^{g} \boldsymbol{U}$ sera noté ${ }^{\mathrm{d}} \boldsymbol{U}$. Enfin, en désignant par $\boldsymbol{I}^{p}\left(\mathfrak{g}, \mathcal{O}_{M}\right)$ le sous $\boldsymbol{U}\left(\mathfrak{g}, \mathcal{O}_{M}\right)$ module de ${ }^{g} \boldsymbol{U}\left(\mathfrak{g}, \mathcal{O}_{M}\right)$ engendré par les $\mathbf{u}_{1}, \cdots, \mathbf{u}_{q}$ avec $q \leqslant p$, on voit que $\boldsymbol{I}^{p}\left(\mathfrak{g}, \mathcal{O}_{M}\right)$ est aussi un sous $\boldsymbol{U}\left(\mathfrak{g}, \mathcal{O}_{M}\right)$-module de ${ }^{\text {ad }} \boldsymbol{U}\left(\mathfrak{g}, \mathcal{O}_{M}\right)$ mais en sus (et surtout!) de ${ }^{g} \boldsymbol{U}\left(\mathfrak{g}, \mathcal{O}_{M}\right)$, i.e. est un idéal à gauche de $\boldsymbol{U}\left(\mathfrak{g}, \mathcal{O}_{M}\right)$. Enfin on posera :

$$
\boldsymbol{I}\left(\mathfrak{g}, \mathcal{O}_{M}\right) \stackrel{\text { def }}{=} \boldsymbol{I}^{1}\left(\mathfrak{g}, \mathcal{O}_{M}\right)=\boldsymbol{U}\left(\mathfrak{g}, \mathcal{O}_{M}\right) \mathfrak{g}
$$

de manière à avoir une suite exacte de $\boldsymbol{U}\left(\mathfrak{g}, \mathcal{O}_{M}\right)$-modules à gauche :

$$
0 \rightarrow \boldsymbol{I}\left(\mathfrak{g}, \mathcal{O}_{M}\right) \rightarrow{ }^{g} \boldsymbol{U}\left(\mathfrak{g}, \mathcal{O}_{M}\right) \stackrel{d_{0}}{\longrightarrow} \mathcal{O}_{M} \rightarrow 0
$$

Dans le même ordre d'idées, on a un exemple plus immédiat en considérant le faisceau des courants à support dans une partie fermée $F \subset M$ qui ne soit pas $G$ invariante. La dérivée de Lie $\mathcal{L}_{\mathbf{u}_{M}^{1,0}}\left(\operatorname{avec} \mathbf{u}^{1,0} \in \mathfrak{g}\right)$ définit la $\boldsymbol{U}\left(\mathfrak{g}, \mathcal{O}_{M}\right)$ structure. (Voir aussi plus bas les $\boldsymbol{U}\left(\mathfrak{g}, \mathcal{O}_{M}\right)$-modules provenant d'une connexion de Chern.)

A vrai dire, via le morphisme de faisceaux d'anneaux : $\boldsymbol{U}\left(\mathfrak{g}, \mathcal{O}_{M}\right) \rightarrow \mathcal{D}_{M}$, tout $\mathcal{D}_{M}$-module est évidemment un $\boldsymbol{U}\left(\mathfrak{g}, \mathcal{O}_{M}\right)$-module. Par cette flèche une action "issue de la base" provient d'ailleurs d'un $\mathcal{D}_{M}$-module.

c) Modules avec une (1,0)-connexion intégrable le long des orbites. Rappelons que l'on a défini dans $[\mathbf{2 1}]$ le morphisme d'algèbre dît "d'évaluation" $\boldsymbol{\Omega}_{M}^{p} \rightarrow \boldsymbol{C}^{p}\left(\mathfrak{g}, \mathcal{O}_{M}\right)$ d'image ${ }^{e v} \boldsymbol{\Omega}_{M}^{p}=\wedge^{p e v} \boldsymbol{\Omega}_{M}^{1}$, au moyen, lorsque $p=1$, de la formule : ${ }^{e v} \omega(\mathbf{u})=\omega\left(\mathbf{u}_{M}\right)$. On appelle faisceau des "costabilisateurs infinitésimaux" le conoyau $\mathcal{Q}$ de la flèche

$$
\text { ev }: \boldsymbol{\Omega}_{M}^{1} \rightarrow \boldsymbol{C}^{1}\left(\mathfrak{g}, \mathcal{O}_{M}\right)
$$

Si $\mathcal{F}$ un $\mathcal{O}_{M}$-module, on dira qu'il possède une $(1,0)$-connexion le long des orbites ( $c f$. aussi [8] à ce sujet) s'il existe une flèche (qui n'est pas $\mathcal{O}_{M}$-linéaire)

$$
\nabla^{\mathfrak{g}}: \mathcal{F} \rightarrow{ }^{e v} \boldsymbol{\Omega}_{M}^{1} \otimes \mathcal{F}
$$

telle que ( $\varphi$ holomorphe) :

$$
\nabla^{\mathfrak{g}}(\varphi \sigma)={ }^{e v} d \varphi \otimes \sigma+\varphi \nabla \sigma
$$


On peut vérifier que cette donnée permet de définir une flèche

$$
\begin{aligned}
\nabla^{\mathfrak{g}}:{ }^{e v} \boldsymbol{\Omega}_{M}^{1} \otimes_{\mathcal{O}_{M}} \mathcal{F} & \longrightarrow{ }^{e v} \Omega_{M}^{2} \otimes_{\mathcal{O}_{M}} \mathcal{F}, \\
\nabla\left({ }^{e v} \omega \otimes \sigma\right) & \stackrel{\text { def }}{=}{ }^{e v}(d \omega) \otimes \sigma-{ }^{e v} \omega \wedge \nabla \sigma .
\end{aligned}
$$

Lorsqu'alors on a $\nabla^{\mathfrak{g}, 2}=0$, on dira que $\mathcal{F}$ possède une $(1,0)$-connexion intégrable le long des orbites. En posant alors : $\left.\boldsymbol{\gamma}_{*}(\mathbf{u}) \sigma=\mathbf{u}_{M}\right\rfloor\left(\nabla^{\mathfrak{g}} \sigma\right)$ (notation de l'annexe section 5 ; observons que la notation $\mathbf{u}\rfloor\left(\nabla^{\mathfrak{g}} \sigma\right)$ aurait aussi un sens et donnerait le même résultat) on peut vérifier que l'on définit bien une structure de $\boldsymbol{U}\left(\mathfrak{g}, \mathcal{O}_{M}\right)$-module sur $\mathcal{F}$.

Notons aussi qu'il arrive quelquefois que la structure de $\mathcal{O}_{M}$-module de $\mathcal{F}$ s'obtienne par restriction à partir d'une structure de $\mathcal{E}_{M}$-module sur $\mathcal{F}$ et que, pour $f$ fonction $\mathcal{C}^{\infty}$, on ait en fait

$$
\nabla^{\mathfrak{g}}(f \sigma)={ }^{e v} \partial f \otimes_{\mathcal{E}_{M}} \sigma+f \nabla^{\mathfrak{g}} \sigma
$$

étant entendu que :

$$
{ }^{e v} \mathcal{E}_{M}^{1,0} \otimes \mathcal{E}_{M} \mathcal{F} \approx{ }^{e v} \Omega_{M}^{1} \otimes \mathcal{O}_{M} \mathcal{F}
$$

dès lors que l'on a posé ${ }^{e v} \mathcal{E}_{M}^{1,0} \stackrel{\text { def }}{=}{ }^{e v} \boldsymbol{\Omega}_{M}^{1} \otimes_{\mathcal{O}_{M}} \mathcal{E}_{M}$; dernier faisceau qui, via la $\mathcal{O}_{M^{-}}$ platitude de $\mathcal{E}_{M}$, est inclus dans $\mathcal{E}_{M}^{1,0}\left(\mathfrak{g}, \mathcal{O}_{M}\right)$.

d) Connexions hermitiennes. Un exemple de cette dernière situation est la suivante : Soit $E \rightarrow M$ un fibré vectoriel holomorphe de rang fini au dessus de $M$ muni d'une métrique hermitienne. Considérons la connexion hermitienne $\nabla=\nabla^{1,0}+\bar{\partial}$. La notation $\nabla$ désignera aussi la différentielle extérieure covariante pour les formes à valeurs dans $E$. La courbure $\nabla^{2}$ est une $(1,1)$-forme à valeurs $\operatorname{End}(E)$, si bien que $\left(\nabla^{1,0}\right)^{2}=0$. Si bien que la flèche composée :

$$
\mathcal{E}_{M}(E) \stackrel{\nabla^{1,0}}{\rightarrow} \boldsymbol{\Omega}_{M}^{1} \otimes_{\mathcal{O}_{M}} \mathcal{E}_{M}(E) \rightarrow{ }^{e v} \boldsymbol{\Omega}_{M}^{1} \otimes_{\mathcal{O}_{M}} \mathcal{E}_{M}(E) \rightarrow 0
$$

permet de définir une $(1,0)$-connexion intégrable le long des orbites. Ainsi, et une fois encore tout à fait indépendamment d'une $G$-action locale sur $E$, la simple donnée d'une métrique permet de définir des opérateurs $\gamma_{*}(\mathbf{u})$ qui vérifient les formules (4). La connexion de Chern se prolonge pour définir un opérateur de (1,0)-dérivée covariante $\mathcal{E}_{M}^{0,1}(E) \stackrel{\nabla^{1,0}}{\rightarrow} \mathcal{E}_{M}^{1,1}(E)$ de telle manière que :

$$
\left.\boldsymbol{\gamma}_{*}(\mathbf{u}) \sigma^{0,1} \stackrel{\text { def }}{=} \mathbf{u}_{M}\right\rfloor \nabla^{1,0} \sigma^{0,1}
$$

fasse de $\mathcal{E}_{M}^{0,1}(E)$ un $\boldsymbol{U}\left(\mathfrak{g}, \mathcal{O}_{M}\right)$-module.

On remarquera alors que, contrairement au cas des structures de $\mathcal{E}_{M}$-modules sur $\mathcal{E}_{M_{M}}(E)$ qui proviendraient d'une $G$-équivariance holomorphe sur $E$, la formule $\boldsymbol{\gamma}_{*}(\mathbf{u}) \bar{\partial}=\bar{\partial} \gamma_{*}(\mathbf{u})$ évidemment fondamentale dans [19], n'est plus vraie comme donc, aussi, le calcul de la cohomologie totale au moyen du complexe des cochaînes totales. Enfin le sous-faisceau $\mathcal{O}_{M}(E) \hookrightarrow \mathcal{E}_{M}(E)$ des germes de sections holomorphes n'est pas, en général (symboles de Christoffel non nécessairement holomorphes), un sous$\boldsymbol{U}\left(\mathfrak{g}, \mathcal{O}_{M}\right)$-module à partir duquel ( $c f$. plus bas) $\mathcal{E}_{M}(E)$ s'obtiendrait par lissification.

e) En faisant, comme plus haut dans "deuxième cas particulier", $\mathbf{V}=U(\mathfrak{g}, \mathbb{C})$, on retrouve $\boldsymbol{U}\left(\mathfrak{g}, \mathcal{O}_{M}\right)$ lui même avec sa structure naturelle de $\boldsymbol{U}\left(\mathfrak{g}, \mathcal{O}_{M}\right)$-module donnée par la multiplication à gauche, i.e. ${ }^{g} \boldsymbol{U}$. 
f) De manière plus générale, si $\mathcal{M}$ est un $\boldsymbol{U}\left(\mathfrak{g}, \mathcal{O}_{M}\right)$-module à gauche et $\mathbf{V}$ comme ci-dessus, $\mathcal{M} \otimes_{\mathbb{C}} \mathbf{V}$ devient un $\boldsymbol{U}\left(\mathfrak{g}, \mathcal{O}_{M}\right)$-module au moyen de $\varphi\left(m \otimes_{\mathbb{C}} \boldsymbol{v}\right)=\varphi m \otimes_{\mathbb{C}} \boldsymbol{v}$ et

$$
\mathbf{u}\left(m \otimes_{\mathbb{C}} \boldsymbol{v}\right)=\mathbf{u} m \otimes_{\mathbb{C}} \boldsymbol{v}+m \otimes_{\mathbb{C}} \mathbf{u} \boldsymbol{v}
$$

g) Indiquons ici, pour finir, ce que l'on pourrait appeler le cas "le plus naturel". Il s'agit des faisceaux de formes différentielles ou de champs de vecteurs, et plus généralement même, de champs de tenseurs, sur la variété $M$ dont les sections peuvent être dérivées au sens de Lie. Ils deviennent des $\boldsymbol{U}\left(\mathfrak{g}, \mathcal{O}_{M}\right)$-modules au moyen de $\gamma_{*}(\mathbf{u})=\mathcal{L}_{\mathbf{u}_{M}}$. Si $\boldsymbol{X}$ est une section locale de $\boldsymbol{E}_{1}\left(\mathfrak{g}, \mathcal{O}_{M}\right)$, on prendra garde que : $\boldsymbol{\gamma}_{*}(\boldsymbol{x}) \neq \mathcal{L}_{\boldsymbol{X}_{M}}$ ne serait ce que parce que $\mathcal{L}_{\varphi \boldsymbol{X}_{M}} \neq \varphi \mathcal{L}_{\boldsymbol{X}_{M}}$, alors que :

$$
\gamma_{*}(\varphi \boldsymbol{X})=\varphi \gamma_{*}(\boldsymbol{X}) .
$$

\subsection{Autres procédés de construction de $\boldsymbol{U}\left(\mathfrak{g}, \mathcal{O}_{M}\right)$-modules.}

Il nous sera utile de généraliser comme suit la réciproque partielle évoquée plus haut. Supposons que $\mathcal{F}$ et $\mathcal{G}$ soient $\operatorname{des} \boldsymbol{U}\left(\mathfrak{g}, \mathcal{O}_{M}\right)$-modules. On rappelle qu'une section globale de $\Phi^{M} \in \operatorname{Hom}_{\mathcal{O}_{M}}(\mathcal{F}, \mathcal{G})$ consiste à se donner, pour tout ouvert $U \subset M$, un homomorphisme de $H^{0}\left(U, \mathcal{O}_{M}\right)$-modules :

$$
\phi_{U} \in \operatorname{Hom}_{H^{0}\left(U, \mathcal{O}_{M}\right)}\left(H^{0}(U, \mathcal{F}), H^{0}(U, \mathcal{G})\right)
$$

avec les compatibilités bien connues relatives à l'inclusion $V \subset U$. Mais alors, les morphismes $\boldsymbol{\gamma}_{*}(\mathbf{u}) \phi_{U}$ donnés par :

$$
\gamma_{*}(\mathbf{u}) \phi_{U} \cdot f_{U}=\mathbf{u} \cdot \phi_{U}\left(f_{U}\right)-\phi_{U}\left(\mathbf{u} \cdot f_{U}\right)
$$

qui sont aisément des $H^{0}\left(U, \mathcal{O}_{M}\right)$-flèches, vérifient clairement les propriétés de compatibilités relatives aux inclusions. On voit donc comment définir une structure de $U(\mathfrak{g}, \mathbb{C})$-module sur $\operatorname{Hom}_{\mathcal{O}_{M}}(\mathcal{F}, \mathcal{G})$ en définissant $\mathbf{u} \cdot \phi$ précisément comme cette famille compatible. (Le lecteur voit même comment mutatis mutandis faire de

$$
\operatorname{Ext}_{\mathcal{O}_{M}}(\mathcal{F}, \mathcal{G})
$$

un $U(\mathfrak{g}, \mathbb{C})$-module.) On en déduit deux nouveaux exemples de $\boldsymbol{U}\left(\mathfrak{g}, \mathcal{O}_{M}\right)$-modules :

1er) $\mathcal{H o m}_{\mathcal{O}_{M}}(\mathcal{F}, \mathcal{G})$. En effet la commutativité de $\mathcal{O}_{M}$ fait de manière naturelle de ce faisceau un $\mathcal{O}_{M}$-module. Rappellons d'abord qu'une section $\Phi^{U}$ de ce faisceau n'est rien d'autre qu'un élément de $\operatorname{Hom}_{\mathcal{O}_{M} \mid U}\left(\mathcal{F}_{\mid U}, \mathcal{G}_{\mid U}\right)$, i.e. une famille de flèches $H^{0}\left(V, \mathcal{O}_{M}\right)$-linéaires :

$$
H^{0}(V, \mathcal{F}) \stackrel{\phi_{V}}{\rightarrow} H^{0}(V, \mathcal{G})
$$

pour tout ouvert $V \subset U$, avec des propriétés classiques de compatibilité. La quantité $\gamma_{*}(\mathbf{u}) \Phi^{U}=\mathbf{u} \cdot \Phi^{U}$ est alors définissable comme la famille des $\boldsymbol{\gamma}_{*}(\mathbf{u}) \phi_{V}$, pour tout ouvert $V \subset U$. On vérifie aisément la relation 5 . On a des propriétés fonctorielles que l'on n'explicite pas. Dans le cas particulier $\mathcal{G}=\mathcal{O}_{M}$, on obtient l'action contragrédiente sur : $\check{\mathcal{F}} \stackrel{\text { def }}{=} \mathcal{H o m}_{\mathcal{O}_{M}}\left(\mathcal{F}, \mathcal{O}_{M}\right)$. Enfin terminons ce 1er) avec l'observation que :

$$
\mathcal{H o m}_{\mathcal{O}_{M}}(\mathcal{F}, \mathcal{G})^{\mathfrak{g}}=\mathcal{H o m}_{U\left(\mathfrak{g}, \mathcal{O}_{M}\right)}(\mathcal{F}, \mathcal{G}) .
$$

2eme) A partir du $U(\mathfrak{g})$-module $\operatorname{Hom}_{\mathcal{O}_{M}}(\mathcal{F}, \mathcal{G})$, on peut encore, comme nous l'avons 
vu plus haut, définir le $\boldsymbol{U}\left(\mathfrak{g}, \mathcal{O}_{M}\right)$-module $\mathcal{O}_{M} \otimes_{\mathbb{C}} \operatorname{Hom}_{\mathcal{O}_{M}}(\mathcal{F}, \mathcal{G})$ ainsi qu'un morphisme de $\boldsymbol{U}\left(\mathfrak{g}, \mathcal{O}_{M}\right)$-module :

$$
\mathcal{O}_{M} \otimes_{\mathbb{C}} \operatorname{Hom}_{\mathcal{O}_{M}}(\mathcal{F}, \mathcal{G}) \rightarrow \mathcal{H o m}_{\mathcal{O}_{M}}(\mathcal{F}, \mathcal{G}) .
$$

3eme) Supposons encore que $\mathcal{F}$ et $\mathcal{G}$ soient $\operatorname{des} \boldsymbol{U}\left(\mathfrak{g}, \mathcal{O}_{M}\right)$-modules à gauche. Par là même ils sont aussi des $\mathcal{O}_{M}$-modules à gauche. Comme il peut arriver que $\mathcal{F}$ possède aussi une structure de $\mathcal{O}_{M}$-module à droite différente de celle donnée à gauche (et qui en fasse éventuellement un $\mathcal{O}_{M}$-bimodule) nous ferons le produit tensoriel (sur $\mathcal{O}_{M}$ ) $\mathcal{F}^{g}{ }_{\mathcal{O}_{M}} \mathcal{G}$ des $\mathcal{O}_{M}$-structures à gauche, i.e. telle que

$$
h \stackrel{g}{\otimes}^{\mathcal{O}_{M}} \tau=\stackrel{g}{\otimes}_{\mathcal{O}_{M}} h \tau=h\left(\sigma^{g} \mathcal{O}_{M} \tau\right)
$$

où $h$ désigne un germe de $\mathcal{O}_{M}$. On définit alors sur ce $\mathcal{O}_{M}$-module une $\mathfrak{g}$-action à gauche (i.e. qui vérifie (5)) en posant :

$$
\mathbf{u} \cdot\left(\sigma^{g} \mathcal{O}_{M} \tau\right)=\mathbf{u} \cdot \sigma^{g} \mathcal{O}_{M} \tau+\sigma^{g} \mathcal{O}_{M} \mathbf{u} \cdot \tau .
$$

Un des grands intérêts de ce produit tensoriel " $\otimes^{g} \mathcal{O}_{M}$ " sur les $\boldsymbol{U}\left(\mathfrak{g}, \mathcal{O}_{M}\right)$-modules à gauche sera de pouvoir formuler un résultat d'adjonction "exotique", i.e. qui n'est point directement la faisceautisation d'un résultat d'adjonction pour les modules sur les anneaux généraux. En effet soient $\mathcal{A}$ et $\mathcal{B}$ et $\mathcal{M}$ trois $\boldsymbol{U}\left(\mathfrak{g}, \mathcal{O}_{M}\right)$-modules à gauche qui soient par là même des $\mathcal{O}_{M}$-modules. On a, par "faisceautisation" la formule d'adjonction "usuelle" :

$$
\Phi: \operatorname{Hom}_{\mathcal{O}_{M}}\left(\mathcal{A} \stackrel{g}{\otimes}_{\mathcal{O}_{M}} \mathcal{B}, \mathcal{M}\right) \approx \operatorname{Hom}_{\mathcal{O}_{M}}\left(\mathcal{A}, \mathcal{H} m_{\mathcal{O}_{M}}(\mathcal{B}, \mathcal{M})\right)
$$

où $\Phi$ est un isomorphisme de $\mathcal{O}_{M}$-modules. Mais on sait par ailleurs, au moyen d'une combinaison des procédés des 1er) et 3eme) immédiatement plus haut, que chacun des membres est aussi un $\boldsymbol{U}\left(\mathfrak{g}, \mathcal{O}_{M}\right)$-modules. Mais alors l'isomorphisme $\Phi$ ci-dessus est $\mathfrak{g}$-équivariant; en particulier, par restriction de $\Phi$ on a un isomorphisme d'adjonction "exotique" :

$$
\operatorname{Hom}_{\boldsymbol{U}\left(\mathfrak{g}, \mathcal{O}_{M}\right)}\left(\mathcal{A}{\stackrel{g}{\otimes} \mathcal{O}_{M}}^{\mathcal{B}}, \mathcal{M}\right) \approx \operatorname{Hom}_{\boldsymbol{U}\left(\mathfrak{g}, \mathcal{O}_{M}\right)}\left(\mathcal{A}, \mathcal{H o m}_{\mathcal{O}_{M}}(\mathcal{B}, \mathcal{M})\right)
$$

ainsi que sa version "faisceautisée"

$$
\mathcal{H o m}_{\boldsymbol{U}\left(\mathfrak{g}, \mathcal{O}_{M}\right)}\left(\mathcal{A} \otimes^{g} \mathcal{O}_{M} \mathcal{B}, \mathcal{M}\right) \approx \mathcal{H o m}_{\boldsymbol{U}\left(\mathfrak{g}, \mathcal{O}_{M}\right)}\left(\mathcal{A}, \mathcal{H o m}_{\mathcal{O}_{M}}(\mathcal{B}, \mathcal{M})\right)
$$

Les démonstrations sont pour l'essentiel des "faisceautisations" que nous n'explicitons pas.

Supposons maintenant que $\mathcal{A}$ soit un sous-faisceau d'anneaux de $\boldsymbol{U}\left(\mathfrak{g}, \mathcal{O}_{M}\right)$; et $\mathcal{N}$ un $\mathcal{A}$-module à gauche; envisageons deux cas :

4a) Par la seule multiplication sur le facteur de gauche, ${ }^{g} \boldsymbol{U}\left(\mathfrak{g}, \mathcal{O}_{M}\right) \otimes_{\mathcal{A}} \mathcal{N}$ devient un $\boldsymbol{U}\left(\mathfrak{g}, \mathcal{O}_{M}\right)$-module à gauche. Étudions spécialement le cas où

$$
\mathcal{A}=\mathcal{O}_{M} \subset \boldsymbol{U}\left(\mathfrak{g}, \mathcal{O}_{M}\right) .
$$

Il est bon d'observer que, compris comme module à droite sur $\mathcal{O}_{M}$, le faisceau 
$\boldsymbol{U}\left(\mathfrak{g}, \mathcal{O}_{M}\right)$ est plat, i.e. le foncteur ${ }^{g} \boldsymbol{U}\left(\mathfrak{g}, \mathcal{O}_{M}\right) \otimes_{\mathcal{O}_{M}}-\operatorname{de} \operatorname{Mod}\left(\mathcal{O}_{M}\right)$ vers

$$
\operatorname{Mod}\left(\boldsymbol{U}\left(\mathfrak{g}, \mathcal{O}_{M}\right)\right)
$$

est exact. Mais ce foncteur est l'adjoint à gauche "usuel" du foncteur "d'oubli"

$$
U: \operatorname{Mod}\left(\boldsymbol{U}\left(\mathfrak{g}, \mathcal{O}_{M}\right)\right) \rightarrow \operatorname{Mod}\left(\mathcal{O}_{M}\right)
$$

qui, à un $\boldsymbol{U}\left(\mathfrak{g}, \mathcal{O}_{M}\right)$-module fait correspondre le $\mathcal{O}_{M}$-module sous-jacent. Mais alors, l'exactitude du foncteur signalée immédiatement plus haut, implique ([12] Theorem $\mathbf{1 2 . 1}$ du chapitre 4) qu'un $\boldsymbol{U}\left(\mathfrak{g}, \mathcal{O}_{M}\right)$-module injectif est aussi injectif en tant que $\mathcal{O}_{M}$-module.

4b) Observons que si $\mathbf{A}$ est un $U(\mathfrak{g}, \mathbb{C})$-module comme dans 2.1. et que

$$
\mathcal{A}=\mathcal{O}_{M} \subset \boldsymbol{U}\left(\mathfrak{g}, \mathcal{O}_{M}\right),
$$

que ${ }^{g} \mathcal{O}_{M} \otimes_{\mathbb{C}} \mathbf{A}$ est distinct de ce que l'on a désigné $\mathcal{O}_{M} \otimes_{\mathbb{C}} \mathbf{A}$ dans 2.1 à moins que la $\mathfrak{g}$-action sur $\mathbf{A}$ ne soit triviale.

5) $\mathcal{E}_{x} t_{\mathcal{O}_{M}}^{p}(\mathcal{F}, \mathcal{G})$. Soient $\mathcal{F}$ et $\mathcal{G} \operatorname{deux} \boldsymbol{U}\left(\mathfrak{g}, \mathcal{O}_{M}\right)$ modules et soient $\mathcal{I} \bullet(\mathcal{G})$ une résolution injective de $\mathcal{G}$ considéré comme objet de $\operatorname{Mod}\left(\boldsymbol{U}\left(\mathfrak{g}, \mathcal{O}_{M}\right)\right)$. Le complexe des $\mathcal{H} m_{\mathcal{O}_{M}}(\mathcal{F}, \mathcal{I} \bullet(\mathcal{G}))$ est donc ( $c f$. le 1er) du présent 2.3. un complexe de $\boldsymbol{U}\left(\mathfrak{g}, \mathcal{O}_{M}\right)$ modules et sa cohomologie (faisceautique) est naturellement un $\boldsymbol{U}\left(\mathfrak{g}, \mathcal{O}_{M}\right)$-module. Mais les $\mathcal{I} \bullet(\mathcal{G})$ étant ( $c f$. 4a plus haut) injectifs en tant qu'objets de $\operatorname{Mod}\left(\mathcal{O}_{M}\right)$, la cohomologie en degré $p$ n'est rien d'autre que $\mathcal{E} x t_{\mathcal{O}_{M}}^{p}(\mathcal{F}, \mathcal{G})$ qui se trouve donc être muni d'une structure de $\boldsymbol{U}\left(\mathfrak{g}, \mathcal{O}_{M}\right)$-module qui ne dépend pas du choix auxiliaire de la résolution injective et qui par "oubli" en fait le $\mathcal{O}_{M}$-module usuel.

6) $\boldsymbol{U}\left(\mathfrak{g}, \mathcal{C}_{M}^{\infty}\right)$-modules. Considérons $\mathcal{C}_{M}^{\infty}$ avec sa structure de $\boldsymbol{U}\left(\mathfrak{g}, \mathcal{O}_{M}\right)$-module définie au 3eme) du 1.1. Evidemment

$$
\boldsymbol{U}\left(\mathfrak{g}, \mathcal{C}_{M}^{\infty}\right) \stackrel{\text { def }}{=} \mathcal{C}_{M}^{\infty} \otimes^{g} \mathcal{O}_{M} \boldsymbol{U}\left(\mathfrak{g}, \mathcal{O}_{M}\right)=\mathcal{C}_{M}^{\infty} \otimes_{\otimes_{\mathbb{C}}} U(\mathfrak{g}, \mathbb{C})
$$

est muni d'une structure de $\boldsymbol{U}\left(\mathfrak{g}, \mathcal{O}_{M}\right)$-module. A vrai dire $\boldsymbol{U}\left(\mathfrak{g}, \mathcal{C}_{M}^{\infty}\right)$ est un faisceau d'anneaux au moyen d'une multiplication, définie de proche en proche, au moyen de la formule de commutation

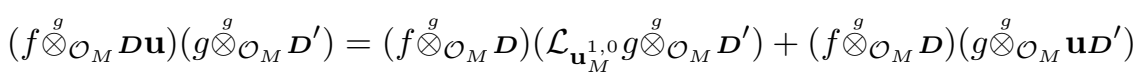

et la structure trouvée plus haut n'est rien d'autre que celle obtenue par la multiplication à gauche au moyen de l'inclusion d'anneaux : $\boldsymbol{U}\left(\mathfrak{g}, \mathcal{O}_{M}\right) \hookrightarrow \boldsymbol{U}\left(\mathfrak{g}, \mathcal{C}_{M}^{\infty}\right)$. On a la notion immédiate de $\boldsymbol{U}\left(\mathfrak{g}, \mathcal{C}_{M}^{\infty}\right)$-module. Si $\mathcal{F}$ est un $\boldsymbol{U}\left(\mathfrak{g}, \mathcal{O}_{M}\right)$-module, le produit tensoriel :

$$
\mathrm{L} \mathcal{F} \stackrel{\text { def }}{=} \boldsymbol{U}\left(\mathfrak{g}, \mathcal{C}_{M}^{\infty}\right) \otimes_{\boldsymbol{U}\left(\mathfrak{g}, \mathcal{O}_{M}\right)} \mathcal{F}
$$

devient, par la multiplication à gauche sur son facteur de gauche, un $\boldsymbol{U}\left(\mathfrak{g}, \mathcal{C}_{M}^{\infty}\right)$ module. En fait il s'agit aussi du $\boldsymbol{U}\left(\mathfrak{g}, \mathcal{O}_{M}\right)$-module $\mathrm{L} \mathcal{F}=\mathcal{C}_{M}^{\infty}{ }^{g} \mathcal{O}_{M} \mathcal{F}$ avec la multiplication de $\mathcal{C}_{M}^{\infty} \subset \boldsymbol{U}\left(\mathfrak{g}, \mathcal{C}_{M}^{\infty}\right)$ sur son facteur de gauche. En sens contraire, tout $\boldsymbol{U}\left(\mathfrak{g}, \mathcal{C}_{M}^{\infty}\right)$-module $\mathcal{G}$ a une structure sous-jacente de $\boldsymbol{U}\left(\mathfrak{g}, \mathcal{O}_{M}\right)$-module par l'inclusion d'anneaux $\boldsymbol{U}\left(\mathfrak{g}, \mathcal{O}_{M}\right) \hookrightarrow \boldsymbol{U}\left(\mathfrak{g}, \mathcal{C}_{M}^{\infty}\right)$. Avec cette structure, on le désignera UG ; ensemblistement c'est exactement $\mathcal{G}$ lui même, aussi dira-t-on de U qu'il est le foncteur d'oubli. Il est bien classique que L est l'adjoint à gauche de U comme il en sera fait usage dans le 3.5. Pour finir, observons ici que si $\psi^{0, q}$ est une $(0, q)$-forme $\mathcal{C}^{\infty}$ locale et $\mathbf{u} \in \mathfrak{g}$, qu'en posant $\left.\boldsymbol{\gamma}_{*}(\mathbf{u}) \psi^{0, q}=\mathbf{u}_{M}\right\rfloor \partial \psi^{0, q}$, où $\left.\mathbf{u}_{M}\right\rfloor$ désigne ( $c f .5$. Annexe) 
le produit intérieur par le (1,0)-champ holomorphe $\mathbf{u}_{M}$, on fait de $\mathcal{E}_{M}^{0, q}$ un $\boldsymbol{U}\left(\mathfrak{g}, \mathcal{C}_{M}^{\infty}\right)$ module qui ne s'obtient pas par le procédé de lissification décrit immédiatement plus haut.

\section{La cohomologie totale comme foncteur dérivé.}

\subsection{Une résolution du $U\left(\mathfrak{g}, \mathcal{O}_{M}\right)$-module $\mathcal{O}_{M}$ "quasiment" projective.}

Soient maintenant $\mathcal{F}$ et $\mathcal{G}$ deux $\boldsymbol{U}\left(\mathfrak{g}, \mathcal{O}_{M}\right)$-modules. On sait $(c f . \quad[\mathbf{1 0}])$ que $\mathcal{G}$ possède dans la catégorie des $\boldsymbol{U}\left(\mathfrak{g}, \mathcal{O}_{M}\right)$ - modules une résolution $\mathcal{I} \bullet(\mathcal{G})$ par des faisceaux qui sont des objets injectifs dans cette catégorie. (Ce qui n'implique pas, $a$ priori, que toute fibre $\mathcal{I}_{M, \boldsymbol{m}}^{\bullet}$ soit un $\mathcal{O}_{M, \boldsymbol{m}} \otimes_{\mathbb{C}} U(\mathfrak{g})$-module injectif). La cohomologie du complexe

$$
\operatorname{Hom}_{\boldsymbol{U}\left(\mathfrak{g}, \mathcal{O}_{M}\right)}(M ; \mathcal{F}, \mathcal{I} \bullet(\mathcal{G}))
$$

est classiquement désignée par $\operatorname{Ext}_{\boldsymbol{U}\left(\mathfrak{g}, \mathcal{O}_{M}\right)}^{\bullet}(M ; \mathcal{F}, \mathcal{G})$; et cet invariant mesure les classes d'équivalence, au sens de Baer, des extensions de $\boldsymbol{U}\left(\mathfrak{g}, \mathcal{O}_{M}\right)$-modules de $\mathcal{F}$ par $\mathcal{G}$. Enfin on a une première suite spectrale qui aboutit vers $\operatorname{Ext}_{\boldsymbol{U}\left(\mathfrak{g}, \mathcal{O}_{M}\right)}^{p+q}(M ; \mathcal{F}, \mathcal{G})$ de deuxième terme

$$
E_{2}^{p, q}=H^{p}\left(M, \mathcal{E} x t_{\boldsymbol{U}\left(\mathfrak{g}, \mathcal{O}_{M}\right)}^{q}(\mathcal{F}, \mathcal{G})\right) .
$$

Il est bon de noter que ceci démontre que $\operatorname{Ext}_{\boldsymbol{U}\left(\mathfrak{g}, \mathcal{O}_{M}\right)}(M ; \mathcal{F}, \mathcal{G})$ est aussi l'hypercohomologie du complexe de faisceaux

$$
\mathcal{H o m}_{\boldsymbol{U}\left(\mathfrak{g}, \mathcal{O}_{M}\right)}\left(\mathcal{F}, \mathcal{I}^{\bullet}(\mathcal{G})\right)
$$

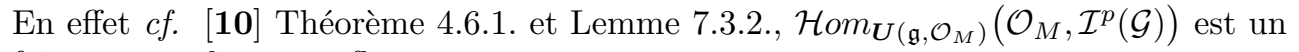
faisceau acyclique car flasque.

En tout état de cause, on comprend l'intérêt d'exprimer la cohomologie faisceautique $\mathcal{E} x t_{\boldsymbol{U}\left(\mathfrak{g}, \mathcal{O}_{M}\right)}^{\bullet}(M ; \mathcal{F}, \mathcal{G})$. Notre premier objet sera, dans le cas où $\mathcal{F}=\mathcal{O}_{M}$, de l'exprimer comme cohomologie faisceautique d'un complexe moins abstrait que celui obtenu au moyen d'une résolution injective de $\mathcal{G}$. Pour cela considérons : $E_{p}(\mathfrak{g}) \stackrel{\text { def }}{=} \wedge^{p} \mathfrak{g}$, le $\boldsymbol{U}\left(\mathfrak{g}, \mathcal{O}_{M}\right)$-module à gauche :

$$
\mathcal{L}_{p} \stackrel{\text { def }}{=}{ }^{g} \boldsymbol{U}\left(\mathfrak{g}, \mathcal{O}_{M}\right) \otimes_{\mathbb{C}} E_{p}(\mathfrak{g})={ }^{g} \boldsymbol{U}\left(\mathfrak{g}, \mathcal{O}_{M}\right) \otimes_{\mathcal{O}_{M}} \boldsymbol{E}_{p}\left(\mathfrak{g}, \mathcal{O}_{M}\right),
$$

s'écrit aussi $\mathcal{L}_{p}=\mathcal{O}_{M} \otimes \mathbf{V}_{M}$; comme dans "deuxième cas particulier" à la toute fin du 2.1., où $\mathbf{V}$ désigne le $U(\mathfrak{g})$-module $\mathbf{V} \stackrel{\text { def }}{=} U(\mathfrak{g}) \otimes_{\mathbb{C}} E_{p}(\mathfrak{g})$ défini par l'action :

$$
\mathbf{D}\left(\mathbf{D}^{\prime} \otimes_{\mathbb{C}} e_{p}\right)=\left(\mathbf{D D}^{\prime}\right) \otimes_{\mathbb{C}} e_{p} .
$$

En particulier, $c f . \quad[\mathbf{1 2}]$, la différentielle

$$
d_{p}: U(\mathfrak{g}) \otimes_{\mathbb{C}} E_{p} \rightarrow U(\mathfrak{g}) \otimes_{\mathbb{C}} E_{p-1},
$$

que l'on sait être $U(\mathfrak{g})$-linéaire, se prolonge évidemment naturellement en une flèche $\mathcal{O}_{M}$-linéaire, $\mathcal{L}_{p} \stackrel{d_{p}}{\rightarrow} \mathcal{L}_{p-1}$, qui est évidemment un $\boldsymbol{U}\left(\mathfrak{g}, \mathcal{O}_{M}\right)$-module. On explicite $d_{p+1}$ 
comme dans [4], en posant :

$$
d_{p+1}\left(f \mathbf{D} \otimes_{\mathbb{C}} \mathbf{u}_{0} \wedge \cdots \wedge \mathbf{u}_{p}\right)
$$

égal à la quantité :

$$
\begin{aligned}
\sum(-1)^{k} f \mathbf{D} \mathbf{u}_{k} \otimes_{\mathbb{C}}\left(\mathbf{u}_{0} \wedge \cdots \widehat{\mathbf{u}_{k}} \cdots \wedge \mathbf{u}_{p}\right) & \\
& +f \mathbf{D} \otimes_{\mathbb{C}} \sum_{k<\ell}(-1)^{k+\ell}\left(\left[\mathbf{u}_{k}, \mathbf{u}_{\ell}\right] \wedge \cdots \widehat{\mathbf{u}_{k}} \cdots \widehat{\mathbf{u}_{\ell}} \cdots \wedge \mathbf{u}_{p}\right),
\end{aligned}
$$

et cette expression montre plus directement encore, que cette différentielle, toujours notée $d_{p}$, est un morphisme de $\boldsymbol{U}\left(\mathfrak{g}, \mathcal{O}_{M}\right)$-module. La tensorisation sur $\mathbb{C}$ conserve l'exactitude et montre pour finir que $\mathcal{L}_{\bullet}$ est une résolution du $\boldsymbol{U}\left(\mathfrak{g}, \mathcal{O}_{M}\right)$-module $\mathcal{O}_{M}$ par des $\boldsymbol{U}\left(\mathfrak{g}, \mathcal{O}_{M}\right)$-modules libres de type finis (ce qui ne signifie pas qu'ils soient des objets projectifs dans la catégorie abélienne des $\boldsymbol{U}\left(\mathfrak{g}, \mathcal{O}_{M}\right)$-modules). Cette résolution est toutefois constituée de faisceaux dont les fibres $\mathcal{L}_{p, \boldsymbol{m}} \operatorname{sont} \operatorname{des} \boldsymbol{U}\left(\mathfrak{g}, \mathcal{O}_{M}\right)_{\boldsymbol{m}}$-modules projectifs. Rappelons que le théorème 7.4.1. de [10] permet alors d'affirmer :

Proposition 3.1. Si $\mathcal{F}$ est un $\boldsymbol{U}\left(\mathfrak{g}, \mathcal{O}_{M}\right)$-module quelconque, alors :

$$
\mathcal{E x t}_{\boldsymbol{U}\left(\mathfrak{g}, \mathcal{O}_{M}\right)}^{p}\left(\mathcal{O}_{M}, \mathcal{F}\right) \approx \mathcal{H}^{p}\left[\mathcal{H}^{\prime} m_{U\left(\mathfrak{g}, \mathcal{O}_{M}\right)}(\mathcal{L} \bullet \mathcal{F})\right]
$$

Observons aussi ici que

$$
\mathcal{H}^{\circ o m_{U}\left(\mathfrak{g}, \mathcal{O}_{M}\right)}(\mathcal{L} \bullet, \mathcal{F}) \approx \mathcal{H}_{\bullet} m_{\mathcal{O}_{M}}\left(\boldsymbol{E} \bullet\left(\mathfrak{g}, \mathcal{O}_{M}\right), \mathcal{F}\right)
$$

n'est rien d'autre que le complexe

$$
C^{\bullet}(\mathfrak{g}, \mathcal{F}) \stackrel{\text { def }}{=} \boldsymbol{C}^{\bullet}(\mathfrak{g}, \mathbb{C}) \otimes \mathcal{F}
$$

des cochaînes totales holomorphes avec précisément la différentielle $D^{1,0}$ définie plus bas en définition/Rappel 3 ( $c f$. [19] et [21]) et dont on a pu désigner la cohomologie faisceautique par

$$
\mathcal{H}_{D^{1,0}}^{\bullet}(\mathcal{F}) \approx H^{\bullet}\left[\mathcal{H o m}_{\boldsymbol{U}\left(\mathfrak{g}, \mathcal{O}_{M}\right)}(\mathcal{L} \bullet, \mathcal{F})\right]=\mathcal{E} x t_{\boldsymbol{U}\left(\mathfrak{g}, \mathcal{O}_{M}\right)}^{p}\left(\mathcal{O}_{M}, \mathcal{F}\right)
$$

Ce complexe des cochaînes totales holomorphes avec précisément la différentielle $D^{1,0}$ est la faisceautisation d'une chose classique. Indiquons que, pour le voir, il suffit de réinterpréter une section locale $\boldsymbol{c}^{p}$ de $\boldsymbol{C}^{p}(\mathfrak{g}, \mathcal{F})$ comme une section locale de

$$
\mathcal{H o m}_{U(\mathfrak{g}, \mathbb{C})}\left(U(\mathfrak{g}, \mathbb{C}) \otimes E_{p}(\mathfrak{g}), \mathcal{F}\right)
$$

et de "faisceautiser" la formule du cobord donnée dans [2] chapitre XIII $\S 8$.

Dans tout ce qui suit, on appellera $p^{\text {eme }}$ faisceau de cohomologie totale du $\boldsymbol{U}\left(\mathfrak{g}, \mathcal{O}_{M}\right)$-module $\mathcal{F}$, le faisceau $\mathcal{E} x t_{\boldsymbol{U}\left(\mathfrak{g}, \mathcal{O}_{M}\right)}^{p}\left(\mathcal{O}_{M}, \mathcal{F}\right)$, même si $\mathcal{F}$ est un $\boldsymbol{U}\left(\mathfrak{g}, \mathcal{O}_{M}\right)$ module qui ne provient pas, comme dans [19], d'une "G-équivariance géométrique". C'est évidemment la proposition 3.3 , donnée plus bas, qui montre que cette définition est précisément une généralisation de celle donnée dans [19]. 


\subsection{La cohomologie totale calculée au moyen des cochaînes totales est un Ext global.}

Par ailleurs, pour un $\boldsymbol{U}\left(\mathfrak{g}, \mathcal{O}_{M}\right)$-module fixé $\mathcal{F}$, il est clair que le foncteur :

$$
\mathcal{G} \mapsto \operatorname{Ext}_{\boldsymbol{U}\left(\mathfrak{g}, \mathcal{O}_{M}\right)}^{n}(M ; \mathcal{F}, \mathcal{G})
$$

est le $n^{\text {eme }}$ foncteur dérivé à droite du foncteur $h^{\mathcal{F}}$, covariant et exact à gauche :

$$
\mathcal{G} \stackrel{h^{\mathcal{F}}}{\mapsto} \operatorname{Ext}_{\boldsymbol{U}\left(\mathfrak{g}, \mathcal{O}_{M}\right)}^{0}(M ; \mathcal{F}, \mathcal{G})
$$

Dans [19], on a donné la définition de la cohomologie totale d'un fibré holomorphe $G$-équivariant. Un tel fibré, nous l'avons vu, est naturellement un faisceau $\mathcal{O}_{M^{-}}$ cohérent $G$-holomorphiquement équivariant $\mathcal{G}$, pour lequel nous pouvons aisément généraliser ces notions. Pour cela, on considère le faisceau $\mathcal{E}_{M}^{0, q}(\mathcal{G}) \stackrel{\text { def }}{=} \mathcal{E}_{M}^{0, q} \otimes_{\mathcal{O}_{M}} \mathcal{G}$ des $(0, q)$-formes à valeurs dans $\mathcal{G}$, qui est lui aussi, (cf. formule (7) au 3eme) de 2.3) un $\boldsymbol{U}\left(\mathfrak{g}, \mathcal{O}_{M}\right)$-module. Généralisant le (b) de 1.1. (qui correspond au cas $\mathcal{G}=\mathcal{O}_{M}$ ) on pose :

$$
\mathcal{E}_{M}^{p, q}(\mathfrak{g}, \mathcal{G}) \stackrel{\text { def }}{=} C^{p}(\mathfrak{g}, \mathbb{C}) \otimes_{\mathbb{C}} \mathcal{E}_{M}^{0, q}(\mathcal{G})
$$

qu'on appelle $(c f$. . [19]) faisceau des $(p, q)$-cochaînes totales à valeurs dans $\mathcal{G}$. On note $\bar{\partial}: \mathcal{E}_{M}^{0, q}(\mathcal{G}) \rightarrow \mathcal{E}_{M}^{0, q+1}(\mathcal{G})$ la différentielle de Dolbeault et $\delta: C^{p}(\mathfrak{g}, \mathbb{C}) \rightarrow C^{p+1}(\mathfrak{g}, \mathbb{C})$ la différentielle de Koszul sur les cochaînes de l'algèbre de Lie complexe, avec $e_{1}, \cdots e_{n}$ comme base auxiliaire, et $e_{*}^{1}, \cdots, e_{*}^{n}$, sa cobase dans $\mathfrak{g}^{*}$. Rappelons alors :

Définition/Rappels 3.2. Soient $\mathrm{C}^{p} \in C^{p}(\mathfrak{g}, \mathbb{C})$ et $\psi^{0, q}$ une $(0, q)$-forme à valeurs dans le $\boldsymbol{U}\left(\mathfrak{g}, \mathcal{O}_{M}\right)$-module $\mathcal{G}$. Alors, en posant :

$$
\begin{aligned}
D^{1,0}\left(\mathrm{C}^{p} \otimes \psi^{0, q}\right) & =\delta \mathrm{C}^{p} \otimes \psi^{0, q}+\sum_{k} e_{*}^{k} \wedge \mathrm{C}^{p} \otimes \boldsymbol{\gamma}_{*}\left(e_{k}\right) \psi^{0, q} \\
\bar{\partial}\left(\mathrm{C}^{p} \otimes \psi^{0, q}\right) & =(-1)^{p} \mathbf{C}^{p} \otimes \bar{\partial} \psi^{0, q}
\end{aligned}
$$

on a (cf. [19]) :

(i) $\left(D^{1,0}\right)^{2}=\bar{\partial}^{2}=D^{1,0} \bar{\partial}+\bar{\partial} D^{1,0}=0$

(ii) Avec les différentielles $D^{1,0}$ et $\bar{\partial}$, la somme bigraduée $\bigoplus \mathcal{E}^{p, q}(\mathfrak{g}, \mathcal{G})$ devient un double complexe et $D=D^{1,0}+\bar{\partial}$ s'appelle (cf. [19]) différentielle totale. De plus $p+q$ s'appelle ( $[\mathbf{1 9}])$ le degré total de $\mathrm{C}^{p} \otimes \psi^{0, q}$.

(iii) La D-cohomologie du complexe double des sections globales sur $M$, s'appelle la cohomologie totale du $\boldsymbol{U}\left(\mathfrak{g}, \mathcal{O}_{M}\right)$-module $\mathcal{G}$. En degré n on la note $\mathbf{H}^{n}(\mathfrak{g}, \mathcal{G})$.

Théorème 3.3. Soient $G, M, \mathcal{G}$, etc... .comme ce qui précède immédiatement. Alors :

(a) la cohomologie totale $\mathbf{H}^{n}(\mathfrak{g}, \mathcal{G})$ est canoniquement (i.e. fonctoriellement) isomorphe à $\operatorname{Ext}_{\boldsymbol{U}\left(\mathfrak{g}, \mathcal{O}_{M}\right)}^{n}\left(M ; \mathcal{O}_{M}, \mathcal{G}\right)$.

(b) En particulier, la cohomologie totale est le foncteur dérivé du foncteur exact à gauche, qui, à $\mathcal{G}$ fait correspondre l'espace $H^{0}\left(\mathfrak{g}, H^{0}(M, \mathcal{G})\right)$ des sections globales $G$-invariantes.

Avant même la démonstration de ce théorème, nous allons montrer un résultat auxiliaire. La résolution $\mathcal{L}_{p}$ ci dessus combinée ( $c f$. plus haut) à la résolution injective 
$\mathcal{I} \bullet(\mathcal{G})$ du $\boldsymbol{U}\left(\mathfrak{g}, \mathcal{O}_{M}\right)$-module $\mathcal{G}$, permettent maintenant de définir un complexe double de faisceaux par

$$
\Phi^{p, q}=\mathcal{H o m}_{\boldsymbol{U}\left(\mathfrak{g}, \mathcal{O}_{M}\right)}\left(\mathcal{L}_{p}, \mathcal{I}^{q}(\mathcal{G})\right) .
$$

La deuxième suite spectrale "faisceautique" de ce double complexe a pour premier terme :

$$
" E_{1}^{q, p}=\mathcal{H}^{p}\left[\mathcal{H o m}_{\boldsymbol{U}\left(\mathfrak{g}, \mathcal{O}_{M}\right)}\left(\mathcal{L}_{p}, \mathcal{I}^{q}(\mathcal{G})\right)\right],
$$

et vaut donc, par le théorème 7.4.1. de $[\mathbf{1 0}]$,

$$
" E_{1}^{q, p}=\mathcal{E} x t_{\boldsymbol{U}\left(\mathfrak{g}, \mathcal{O}_{M}\right)}^{p}\left(\mathcal{O}_{M}, \mathcal{I}^{q}(\mathcal{G})\right) .
$$

Par ailleurs, et bien que l'injectivité d'un $\boldsymbol{U}\left(\mathfrak{g}, \mathcal{O}_{M}\right)$-module n'implique pas a priori celle de ses fibres, on sait toutefois par [10] qu'elle implique la nullité de cette dernière quantité pour $p \neq 0$. Cela montre en fait :

Lemme 3.4. Avec les notations qui précèdent:

(i) La flèche naturelle:

$$
\mathcal{H o m}_{\boldsymbol{U}\left(\mathfrak{g}, \mathcal{O}_{M}\right)}\left(\mathcal{O}_{M}, \mathcal{I}^{\bullet}(\mathcal{G})\right) \rightarrow \mathcal{H o m}_{\boldsymbol{U}\left(\mathfrak{g}, \mathcal{O}_{M}\right)}\left(\mathcal{L} \bullet, \mathcal{I}^{\bullet}(\mathcal{G})\right)
$$

du complexe "simple" dans le complexe double est un quasi-isomorphisme de complexes de faisceaux.

(ii) En conséquence l'hypercohomologie, en degré $n$, du complexe double est précisément $\operatorname{Ext}_{\boldsymbol{U}\left(\mathfrak{g}, \mathcal{O}_{M}\right)}^{n}\left(M ; \mathcal{O}_{M}, \mathcal{G}\right)$.

Démonstration. Pour montrer (i) il suffit en effet, puisque ${ }^{q} E_{1}^{q, p}=0$ pour $p>0$, de montrer que

$$
" E_{1}^{q, 0}=\mathcal{H o m}_{\boldsymbol{U}\left(\mathfrak{g}, \mathcal{O}_{M}\right)}\left(\mathcal{O}_{M}, \mathcal{I}^{q}(\mathcal{G})\right) .
$$

Or, d'après l'exactitude de

$$
\cdots \rightarrow \mathcal{L}_{1} \rightarrow \mathcal{L}_{0} \rightarrow \mathcal{O}_{M} \rightarrow 0
$$

combinée à l'exactitude du foncteur $\mathcal{H o m}_{\boldsymbol{U}\left(\mathfrak{g}, \mathcal{O}_{M}\right)}\left(-, \mathcal{I}^{q}(\mathcal{G})\right)$ cf. $\quad[\mathbf{1 0}]$ ibidem, on a

$$
" E_{1}^{q, 0}=\mathcal{H o m}_{\boldsymbol{U}\left(\mathfrak{g}, \mathcal{O}_{M}\right)}\left(\mathcal{O}_{M}, \mathcal{I}^{q}(\mathcal{G})\right.
$$

ce qui achève de montrer le (i) du lemme 3.4 ; le (ii) en résultant alors immédiatement.

Démonstration du théorème 3.3. Nous allons maintenant chercher un autre complexe "simple" lui aussi quasi-isomorphe au complexe double. En effet la première suite spectrale faisceautique a pour premier terme :

$$
{ }^{{ }^{\prime}} E_{1}^{p, q}=\mathcal{H}^{q}\left[\mathcal{H o m}_{\boldsymbol{U}\left(\mathfrak{g}, \mathcal{O}_{M}\right)}\left(\mathcal{L}_{p}, \mathcal{I}^{\bullet}(\mathcal{G})\right)\right]=\mathcal{E} x t_{\boldsymbol{U}\left(\mathfrak{g}, \mathcal{O}_{M}\right)}^{q}\left(\mathcal{L}_{p}, \mathcal{G}\right) .
$$

Mais alors $\mathcal{L}_{p}$ étant libre de type fini, le lemme 7.4.1 de [10] montre que ${ }^{`} E_{1}^{p, q}=0$ 
dès que $q>0$, et donc que cette autre suite spectrale dégénère et que le complexe "simple"

$$
E_{1}^{\bullet, 0}=\mathcal{H}^{\circ} m_{\boldsymbol{U}\left(\mathfrak{g}, \mathcal{O}_{M}\right)}\left(\mathcal{L}_{\bullet}, \mathcal{G}\right)
$$

avec la différentielle qui provient de $d_{n}$ s'injecte alors quasi-isomorphiquement dans le complexe double $\Phi^{p, q}$, a donc aussi pour hypercohomologie

$$
\operatorname{Ext}_{\boldsymbol{U}\left(\mathfrak{g}, \mathcal{O}_{M}\right)}^{\bullet}\left(M ; \mathcal{O}_{M}, \mathcal{G}\right) .
$$

Avant de donner une expression plus connue du complexe de faisceaux $E_{1}^{\bullet, 0}$, rappelons que $\mathcal{E}_{M}^{0, \bullet} \otimes_{\mathcal{O}_{M}} \mathcal{G}$ est une résolution fine (ce qui, brièvement se voit, via la platitude de $\mathcal{E}_{M}^{0, q}$ sur $\mathcal{O}_{M}$, par la suite spectrale 5.5.1 de [10], où $\mathcal{G}$ est compris comme un complexe avec un seul terme non nul, en zéro) de $\mathcal{G}$ qui est en fait même une résolution de $\boldsymbol{U}\left(\mathfrak{g}, \mathcal{O}_{M}\right)$-module. Cela permet classiquement de définir à homotopie près, $c f$. [12] "Theorem 4.4", un morphisme de complexes de $\boldsymbol{U}\left(\mathfrak{g}, \mathcal{O}_{M}\right)$-modules :

$$
\mathcal{E}^{0, \bullet} \otimes_{\mathcal{O}_{M}} \mathcal{G} \longrightarrow \mathcal{I}^{\bullet}(\mathcal{G}) \text {. }
$$

Mais alors on a un morphisme de complexes doubles de faisceaux :

$$
\mathcal{H o m}_{\boldsymbol{U}\left(\mathfrak{g}, \mathcal{O}_{M}\right)}\left(\mathcal{L}_{p}, \mathcal{E}^{0, \bullet}(\mathcal{G})\right) \rightarrow \mathcal{H o m}_{\boldsymbol{U}\left(\mathfrak{g}, \mathcal{O}_{M}\right)}\left(\mathcal{L}_{p}, \mathcal{I}^{\bullet}(\mathcal{G})\right) .
$$

Or $\mathcal{H o m}_{\boldsymbol{U}\left(\mathfrak{g}, \mathcal{O}_{M}\right)}\left(\mathcal{L}_{p}, \mathcal{E}^{0, q}(\mathcal{G})\right)$ n'est rien d'autre que l'espace

$$
C^{p}(\mathfrak{g}, \mathbb{C}) \otimes_{\mathbb{C}} \mathcal{E}^{0, q}(\mathcal{G})
$$

des $(p, q)$-cochaînes à valeurs dans le faisceau analytique cohérent "localement $G$ équivariant" $\mathcal{G}$ muni de la différentielle $D$ de $[\mathbf{1 9}]$ définie plus haut en 3.2. On sait (cf. [16]) que le complexe de faisceaux $C^{\bullet}(\mathfrak{g}, \mathbb{C}) \otimes_{\mathbb{C}} \mathcal{G}$ avec la différentielle $D^{1,0}$ de 3.2 se plonge dans ce double complexe et lui est quasi-isomorphe, car en fait, le premier terme ${ }^{`} E_{1}^{p, q}$ de la première suite spectrale faisceautique est nul pour $q>0$ et ${ }^{`} E_{1}^{\bullet, 0}$ avec la différentielle " $d_{1}$ " s'identifie respectivement au complexe précédent muni de $D^{1,0}$; comme de plus tous les faisceaux de ce double complexe sont fins, son hypercohomologie est simplement la $D$-cohomologie de l'espace de ses sections globales $C^{p}(\mathfrak{g}) \otimes_{\mathbb{C}} \mathcal{E}^{0, \bullet}(M, \mathcal{G})$ qui est précisément la cohomologie totale du faisceau localement $G$-équivariant $\mathcal{G}$. Ceci dit, le morphisme de double complexe de faisceaux :

$$
\mathcal{H o m}_{\boldsymbol{U}\left(\mathfrak{g}, \mathcal{O}_{M}\right)}\left(\mathcal{L}_{p}, \mathcal{E}^{0, \bullet}(\mathcal{G})\right) \rightarrow \mathcal{H} \operatorname{Hom}_{\boldsymbol{U}\left(\mathfrak{g}, \mathcal{O}_{M}\right)}\left(\mathcal{L}_{p}, \mathcal{I}^{\bullet}(\mathcal{G})\right)
$$

envoie la première suite spectrale faisceautique vers la première suite spectrale faisceautique. A partir du premier terme, on sait que ne subsistent que les termes

$$
\mathfrak{E}_{1}^{\bullet, 0}=\mathcal{H}^{\circ} m_{\boldsymbol{U}\left(\mathfrak{g}, \mathcal{O}_{M}\right)}\left(\mathcal{L}_{\bullet}, \mathcal{G}\right)=C^{\bullet}(\mathfrak{g}) \otimes_{\mathbb{C}} \mathcal{G}
$$

avec la même différentielle; et cet isomorphisme au niveau du premier terme suffit à démontrer que le $n^{\text {eme }}$ groupe d'hypercohomologie du premier complexe qui est précisément $\mathbf{H}^{n}(\mathfrak{g}, \mathcal{G})$ ( $c f$. 3.2) est égal au $n^{\text {eme }}$ du second, qui, nous l'avons vu plus haut, vaut $\operatorname{Ext}_{\boldsymbol{U}\left(\mathfrak{g}, \mathcal{O}_{M}\right)}^{n}\left(M ; \mathcal{O}_{M}, \mathcal{G}\right)$.

\subsection{Approche au moyen de la composition des foncteurs.}

Soit maintenant $\mathcal{J}$ un faisceau injectif en tant que $\boldsymbol{U}\left(\mathfrak{g}, \mathcal{O}_{M}\right)$-module. Ce qui est différent que de dire que les modules de germes sont injectifs. $H^{0}(M, \mathcal{J})$ est naturellement un $U(\mathfrak{g})$-module dont nous nous proposons de montrer l'injectivité. 
Soient $0 \rightarrow \mathbf{A} \stackrel{u}{\rightarrow} \mathbf{B}$ une suite exacte de $U(\mathfrak{g})$-module et

$$
f: \mathbf{A} \longrightarrow H^{0}(M, \mathcal{J})
$$

un morphisme de $U(\mathfrak{g})$-modules. Il faut trouver une flèche $U(\mathfrak{g})$-linéaire

$$
g: \mathbf{B} \rightarrow H^{0}(M, \mathcal{J}),
$$

telle que l'on ait la factorisation : $f=g u$.

Le procédé donné dans le "deuxième cas particulier" au bas de la définition 2.1, permet, au moyen du foncteur $\mathcal{O}_{M} \otimes_{\mathbb{C}^{-}}$, d'obtenir des $\boldsymbol{U}\left(\mathfrak{g}, \mathcal{O}_{M}\right)$-modules à partir de ces $U(\mathfrak{g})$-modules. On a, ipso-facto, deux morphismes de $\boldsymbol{U}\left(\mathfrak{g}, \mathcal{O}_{M}\right)$-modules

$$
\mathcal{O}_{M} \otimes_{\mathbb{C}} \mathbf{A} \longrightarrow \mathcal{O}_{M} \otimes_{\mathbb{C}} \mathbf{B}
$$

et

$$
\mathcal{O}_{M} \otimes_{\mathbb{C}} \mathbf{A} \longrightarrow \mathcal{O}_{M} \otimes_{\mathbb{C}} H^{0}(M, \mathcal{J}),
$$

le premier $\left(\mathcal{O}_{M}\right.$ plat sur $\left.\mathbb{C}\right)$ évidemment injectif.

La flèche $\boldsymbol{U}\left(\mathfrak{g}, \mathcal{O}_{M}\right)$-linéaire $\mathcal{O}_{M} \otimes_{\mathbb{C}} H^{0}(M, \mathcal{J}) \rightarrow \mathcal{J}$ permet, par composition, de définir un morphisme de $\boldsymbol{U}\left(\mathfrak{g}, \mathcal{O}_{M}\right)$-modules : $\mathcal{O}_{M} \otimes_{\mathbb{C}} \mathbf{A} \rightarrow \mathcal{J}$ et la définition même de l'injectivité, montre que ce dernier morphisme à valeurs dans $\mathcal{J}$, se factorise par le morphisme : $\mathcal{O}_{M} \otimes_{\mathbb{C}} \mathbf{A} \rightarrow \mathcal{O}_{M} \otimes_{\mathbb{C}} \mathbf{B}$. On voit que :

1er) $f$ est la composée des flèches $U(\mathfrak{g})$-linéaires :

$$
\mathbf{A} \subset H^{0}\left(M, \mathcal{O}_{M} \otimes_{\mathbb{C}} \mathbf{A}\right) \rightarrow H^{0}(M, \mathcal{J}) .
$$

2eme) par la première des deux applications $U(\mathfrak{g})$-linéaires :

$$
H^{0}\left(M, \mathcal{O}_{M} \otimes_{\mathbb{C}} \mathbf{A}\right) \rightarrow H^{0}\left(M, \mathcal{O}_{M} \otimes_{\mathbb{C}} \mathbf{B}\right) \rightarrow H^{0}(M, \mathcal{J}),
$$

le sous-espace $\mathbf{A} \subset H^{0}\left(M, \mathcal{O}_{M} \otimes_{\mathbb{C}} \mathbf{A}\right)$ est envoyé sur $\mathbf{B} \subset H^{0}\left(M, \mathcal{O}_{M} \otimes_{\mathbb{C}} \mathbf{B}\right)$ précisément par l'application $u$.

Désignons maintenant par $g$ la restriction de la flèche

$$
H^{0}\left(M, \mathcal{O}_{M} \otimes_{\mathbb{C}} \mathbf{B}\right) \longrightarrow H^{0}(M, \mathcal{J})
$$

au $U(\mathfrak{g})$ sous-module $\mathbf{B} \subset H^{0}\left(M, \mathcal{O}_{M} \otimes_{\mathbb{C}} \mathbf{B}\right)$. On a alors aisément l'égalité recherchée $f=g u$, qui permet d'énoncer :

Proposition 3.5. (i) Soit $\mathcal{J}$ un $\boldsymbol{U}\left(\mathfrak{g}, \mathcal{O}_{M}\right)$-module injectif; alors $H^{0}(M, \mathcal{J})$ est un $U(\mathfrak{g})$-module injectif.

(ii) En conséquence la cohomologie totale:

$$
\mathbf{H}^{n}(\mathfrak{g}, \mathcal{G})=\operatorname{Ext}_{\boldsymbol{U}\left(\mathfrak{g}, \mathcal{O}_{M}\right)}^{n}\left(M ; \mathcal{O}_{M}, \mathcal{G}\right)
$$

est approchée par une suite spectrale dont le deuxième terme est

$$
E_{2}^{p, q}=H^{p}\left(\mathfrak{g}, H^{q}(M, \mathcal{G})\right) .
$$

$A$ un isomorphisme canonique près, cette suite spectrale n'est rien d'autre que celle donnée dans [19] par un tout autre moyen.

Démonstration. On a déjà vu le (i). Par ailleurs le foncteur $h^{\mathcal{O}_{M}}$ du 3.2. qui précède est le composé du premier foncteur additif exact à gauche $\mathcal{G} \rightarrow H^{0}(M, \mathcal{G})$ de 
la catégorie des $\boldsymbol{U}\left(\mathfrak{g}, \mathcal{O}_{M}\right)$-modules vers celle des $U(\mathfrak{g})$-modules (qui sont chacune abéliennes avec "suffisamment" d'objets injectifs) et du foncteur additif exact à gauche qui envoie chaque $U(\mathfrak{g})$-module sur son sous-espace vectoriel complexe des éléments g-invariants. Le (ii) n'est alors qu'une application directe du théorème de Grothendieck sur les dérivés d'un foncteur composé.

Ce résultat permet de généraliser la définition de la cohomologie totale au dessus des espaces singuliers, et même non réduits, sans faire usages des objets $\mathcal{C}^{\infty}$, qui admettent des définitions multiples et non équivalentes entre elles.

\subsection{Analogies avec les $\mathcal{D}$-modules.}

Soit $E$ un $\mathcal{D}_{M}$-module (par exemple un fibré holomorphe "localement plat", c'està-dire avec une connexion holomorphe intégrable); on a vu(bas du b)du 2.2) qu'il était alors naturellement un $\boldsymbol{U}\left(\mathfrak{g}, \mathcal{O}_{M}\right)$-module (dans l'exemple donné immédiatement plus haut, il le devient avec l' action "issue de la base".) Le morphisme $\boldsymbol{e} \boldsymbol{v}$ défini au c) du 2.2. plus haut se généralise pour définir un morphisme

$$
\boldsymbol{\Omega}_{M}^{\bullet} \otimes_{\mathcal{O}_{M}} E \stackrel{e v}{\rightarrow} C^{\bullet}(\mathfrak{g}, E) .
$$

En fait, via la différentielle extérieure covariante, ici de carré nul, et que nous désignerons par $\partial, \boldsymbol{e} \boldsymbol{v}$ devient un morphisme du premier complexe vers le deuxième muni de $D^{1,0}$. On envoie donc l'hypercohomologie du premier complexe (qui est la cohomologie de de Rham) vers celle du second (qui est la cohomologie totale). Indiquons ici brièvement, comment l'interprétation de la cohomologie totale comme foncteur dérivé donné plus haut permet de retrouver ce résultat.

A cette fin rappelons que le $p^{\text {eme }}$ groupe de cohomologie de de Rham de $E$ n'est en fait rien d'autre que $\operatorname{Ext}_{\mathcal{D}_{M}}^{p}\left(M ; \mathcal{O}_{M}, E\right)$, ce qui veut dire qu'elle s'obtient en considérant une résolution injective $\mathcal{I}^{\bullet}$ du $\mathcal{D}_{M}$-module $E$. Le $p^{\text {eme }}$-groupe de cohomologie de de Rham de $E$ n'est alors rien d'autre que le $p^{e m e}$-groupe de cohomologie du complexe $\operatorname{Hom}_{\mathcal{D}_{M}}\left(M ; \mathcal{O}_{M}, \mathcal{I} \bullet\right)$ des "sections globales localement constantes" de $\mathcal{I}^{\bullet}$. Mais, via la flèche : $\boldsymbol{U}\left(\mathfrak{g}, \mathcal{O}_{M}\right) \rightarrow \mathcal{D}_{M}$, on voit que $\mathcal{I}^{\bullet}$ devient un complexe de $\boldsymbol{U}\left(\mathfrak{g}, \mathcal{O}_{M}\right)$-module qui, en tant que telle, n'est plus nécessairement injectif. On sait, par contre, qu'existe un quasi-isomorphisme de complexe de $\boldsymbol{U}\left(\mathfrak{g}, \mathcal{O}_{M}\right)$-modules : $\mathcal{I}^{\bullet} \rightarrow \mathcal{J}^{\bullet}$ où $\mathcal{J}^{\bullet}$ est constitué de $\boldsymbol{U}\left(\mathfrak{g}, \mathcal{O}_{M}\right)$-modules injectifs. La composée des morphismes de complexes :

$$
\operatorname{Hom}_{\mathcal{D}_{M}}\left(M ; \mathcal{O}_{M}, \mathcal{I}^{\bullet}\right) \rightarrow \operatorname{Hom}_{\boldsymbol{U}\left(\mathfrak{g}, \mathcal{O}_{M}\right)}\left(M ; \mathcal{O}_{M}, \mathcal{I}^{\bullet}\right) \rightarrow \operatorname{Hom}_{\boldsymbol{U}\left(\mathfrak{g}, \mathcal{O}_{M}\right)}\left(M ; \mathcal{O}_{M}, \mathcal{J}^{\bullet}\right)
$$

permet alors, via la définition de la cohomologie totale de $E$ comme Ext global donnée au a) du théorème 3.3, de voir que la flèche $\boldsymbol{e} \boldsymbol{v}$, construite dans [21] par des moyens "concrets", et qui envoie la cohomologie de de Rham vers la cohomologie totale n'est qu'une manifestation immanente de la flèche $\boldsymbol{U}\left(\mathfrak{g}, \mathcal{O}_{M}\right) \rightarrow \mathcal{D}_{M}$.

Donnons aussi des indications sur les analogies et leurs limites entre $\boldsymbol{U}\left(\mathfrak{g}, \mathcal{O}_{M}\right)$ et $\mathcal{D}_{M}$-modules. De même que la résolution "quasi projective" du $\boldsymbol{U}\left(\mathfrak{g}, \mathcal{O}_{M}\right)$-module à gauche $\mathcal{O}_{M}$ donnée en 3.1. est l'analogue d'une résolution "quasi projective" connue 
du $\mathcal{D}_{M}$-module à gauche $\mathcal{O}_{M}$, on va chercher l'analogue de la résolution " $\mathcal{D}_{M}$-quasi projective" :

$$
\ldots \stackrel{d}{\rightarrow} \boldsymbol{\Omega}_{M}^{p} \otimes_{\mathcal{O}_{M}} \mathcal{D}_{M} \stackrel{d}{\rightarrow} \boldsymbol{\Omega}_{M}^{p+1} \otimes_{\mathcal{O}_{M}} \mathcal{D}_{M} \stackrel{d}{\rightarrow} \cdots \stackrel{d}{\rightarrow} \boldsymbol{\Omega}_{M}^{m} \otimes_{\mathcal{O}_{M}} \mathcal{D}_{M} \stackrel{d}{\rightarrow} \boldsymbol{\Omega}_{M}^{m} \rightarrow 0
$$

du fibré canonique $\boldsymbol{\Omega}_{M}^{m}$ naturellement considéré comme un $\mathcal{D}_{M}$-module à droite.

Rappelons que le morphisme d'augmentation est donné par $a(\omega \otimes \boldsymbol{D})=\omega \boldsymbol{D}$ et la différentielle, localement par

$$
d\left(\omega^{p} \otimes \boldsymbol{D}\right)=d \omega^{p} \otimes \boldsymbol{D}+\sum_{k=1}^{k=m} d z^{k} \wedge \omega^{p} \otimes \frac{\partial}{\partial z^{k}} \boldsymbol{D} .
$$

L'exactitude de ce complexe se fait par une démonstration bien connue. Nous allons adapter cet énoncé et nous en ferons une démonstration en "retroussant" la résolution "quasi-projective" de $\mathcal{O}_{M}$ donnée plus haut. Pour cela on fait donc de $C^{n}\left(\mathfrak{g}, \mathcal{O}_{M}\right)$ un $\boldsymbol{U}\left(\mathfrak{g}, \mathcal{O}_{M}\right)$-module à droite au moyen de "l'action miroir" de l'action à gauche $\boldsymbol{\gamma}_{*}$, i.e. si $\omega^{n}$ en est une section locale et en utilisant les notations de l'annexe brève 5 pour désigner le produit intérieur, en posant $\left.: \omega^{n} \cdot \mathbf{u}=-D^{1,0} \mathbf{u}\right\rfloor \omega^{n}$.

Fixons d'ailleurs, et pour tout ce qui suivra, un générateur vol $\operatorname{vg}_{\mathfrak{g}}$ non nul dans $\wedge^{n} \mathfrak{g}^{*}$. On voit que :

$$
\left(\varphi \operatorname{vol}_{\mathfrak{g}} \cdot \mathbf{u}=\left(\varphi \triangle \mathbf{u}-\mathbf{u}_{M} \cdot \varphi\right) \operatorname{vol}_{\mathfrak{g}}\right.
$$

Au moyen de la propriété universelle, on commence par observer que la "transposée tordue"

$$
\mathbf{u} \mapsto \mathbf{u}^{\top+\triangle} \stackrel{\text { def }}{=} \triangle \mathbf{u}-\mathbf{u}
$$

(qui ne conserve pas $\mathfrak{g}$ ) définit bien ( $c f$. aussi [9]) un anti-isomorphisme de $\boldsymbol{U}\left(\mathfrak{g}, \mathcal{O}_{M}\right)$ dans lui-même par :

$$
\left(\varphi \mathbf{u}_{1} \cdots \mathbf{u}_{p}\right)^{\top+\triangle}=\left(\triangle \mathbf{u}_{p}-\mathbf{u}_{p}\right)\left(\triangle \mathbf{u}_{p-1}-\mathbf{u}_{p-1}\right) \cdots\left(\triangle \mathbf{u}_{1}-\mathbf{u}_{1}\right) \varphi .
$$

Considérons alors une section locale $\boldsymbol{D} \otimes V_{p}$ du $\boldsymbol{U}\left(\mathfrak{g}, \mathcal{O}_{M}\right)$-module

$$
\mathcal{L}_{p}=\boldsymbol{U}\left(\mathfrak{g}, \mathcal{O}_{M}\right) \otimes E_{p}(\mathfrak{g}, \mathbb{C}) .
$$

Pour tout $p$ compris entre 1 et $n$, on définit

$$
\left.T\left(\boldsymbol{D} \otimes V_{p}\right) \stackrel{\text { def }}{=} V_{p}\right\rfloor \operatorname{vol}_{\mathfrak{g}} \otimes \boldsymbol{D}^{\top+\Delta}
$$

qui est une section locale de

$$
\boldsymbol{C}^{n-p}\left(\mathfrak{g}, \boldsymbol{U}\left(\mathfrak{g}, \mathcal{O}_{M}\right)\right)=\boldsymbol{C}^{n-p}\left(\mathfrak{g}, \mathcal{O}_{M}\right) \otimes_{\mathcal{O}_{M}} \boldsymbol{U}\left(\mathfrak{g}, \mathcal{O}_{M}\right) .
$$

Commençons alors par rappeler que $d_{p}\left(\boldsymbol{D} \otimes V_{p}\right)$ est aussi donné par l'expression :

$$
d_{p}\left(\boldsymbol{D} \otimes V_{p}\right)=\sum_{k=1}^{k=n} \boldsymbol{D} e_{k} \otimes V_{p}\left[e_{*}^{k}-\frac{1}{2} \sum_{1 \leqslant i, j \leqslant n} \boldsymbol{D} \otimes\left[e_{i}, e_{j}\right] \wedge\left(V_{p}\left\lfloor e_{*}^{i} \wedge e_{*}^{j}\right) .\right.\right.
$$


Si bien qu'au moyen de (13) de l'annexe 5 plus bas :

$$
\begin{array}{r}
T d_{p}\left(\boldsymbol{D} \otimes V_{p}\right)=\sum_{k=1}^{k=n}\left(V_{p}\left\lfloor e_{*}^{k}\right)\right\rfloor \operatorname{vol}_{\mathfrak{g}} \otimes\left(\triangle e_{k}-e_{k}\right) \boldsymbol{D}^{\top+\Delta} \\
-\frac{1}{2} \sum_{1 \leqslant i, j \leqslant n}\left(\left[e_{i}, e_{j}\right] \wedge\left(V_{p}\left\lfloor e_{*}^{i} \wedge e_{*}^{j}\right)\right)\right\rfloor \operatorname{vol}_{\mathfrak{g}} \otimes \boldsymbol{D}^{\top+\triangle} \\
\left.=(-1)^{p+1} \sum_{k=1}^{k=n} e_{*}^{k} \wedge V_{p}\right\rfloor \operatorname{vol}_{\mathfrak{g}} \otimes\left(\triangle e_{k}-e_{k}\right) \boldsymbol{D}^{\top+\Delta} \\
\left.-\frac{1}{2}(-1)^{p-2} \sum_{1 \leqslant i, j \leqslant n}\left[e_{i}, e_{j}\right]\right\rfloor\left(\left(V_{p}\left\lfloor e_{*}^{i} \wedge e_{*}^{j}\right)\right\rfloor \operatorname{vol}_{\mathfrak{g}}\right) \otimes \boldsymbol{D}^{\top+\Delta} \\
=(-1)^{p}\left[-\triangle \wedge T\left(\boldsymbol{D} \otimes V_{p}\right)+\sum_{k=1}^{k=n} e_{*}^{k} \wedge V_{p}\right\rfloor \operatorname{vol}_{\mathfrak{g}} \otimes e_{k} \boldsymbol{D}^{\top+\triangle} \\
\left.-\frac{1}{2} \sum_{1 \leqslant i, j \leqslant n}\left[e_{i}, e_{j}\right]\right\rfloor\left(\left(V_{p}\left\lfloor e_{*}^{i}\left\lfloor e_{*}^{j}\right)\right\rfloor \operatorname{vol}_{\mathfrak{g}}\right) \otimes \boldsymbol{D}^{\top+\Delta}\right]
\end{array}
$$

La formule (13) de l'annexe section 5 affirme :

$$
\begin{gathered}
\left.-\frac{1}{2} \sum_{1 \leqslant i, j \leqslant n}\left[e_{i}, e_{j}\right]\right\rfloor\left(\left(V_{p}\left\lfloor e_{*}^{i}\left\lfloor e_{*}^{j}\right)\right\rfloor \operatorname{vol}_{\mathfrak{g}}\right)=-(-1)^{p} \frac{1}{2} \sum_{1 \leqslant i, j \leqslant n}\left[e_{i}, e_{j}\right]\right\rfloor\left(e_{*}^{j} \wedge\left(V_{p}\left\lfloor e_{*}^{i}\right)\right\rfloor \operatorname{vol}_{\mathfrak{g}}\right) \\
\left.=\frac{1}{2} \sum_{1 \leqslant i, j \leqslant n}\left[e_{i}, e_{j}\right]\right\rfloor\left(e_{*}^{j} \wedge e_{*}^{i} \wedge\left(V_{p}\right\rfloor \operatorname{vol}_{\mathfrak{g}}\right) \\
\left.\left.\left.\left.=\frac{1}{2} \sum_{1 \leqslant i, j \leqslant n}\left(\left[e_{i}, e_{j}\right]\right\rfloor e_{*}^{j} \wedge e_{*}^{i}\right) \wedge V_{p}\right\rfloor \operatorname{vol}_{\mathfrak{g}}-\frac{1}{2} \sum_{1 \leqslant i, j \leqslant n} e_{*}^{i} \wedge e_{*}^{j} \wedge\left[e_{i}, e_{j}\right]\right\rfloor\left(V_{p}\right\rfloor \operatorname{vol}_{\mathfrak{g}}\right) .
\end{gathered}
$$

Mais, si on considère les constantes de structure

$$
\left[e_{i}, e_{j}\right]=\sum_{k} c_{i}{ }^{k}{ }_{j} e_{k},
$$

on voit aisément que la 1-cochaîne

$$
\left.\frac{1}{2} \sum_{1 \leqslant i, j \leqslant n}\left[e_{i}, e_{j}\right]\right\rfloor\left(e_{*}^{j} \wedge e_{*}^{i}\right)
$$

vaut $\sum_{i, k} c_{i}{ }_{k} e_{*}^{i}=\triangle$. Quant à l'opérateur

$$
\left.-\frac{1}{2}\left(e_{*}^{i} \wedge e_{*}^{j}\right) \wedge\left[e_{i}, e_{j}\right]\right\rfloor
$$

d'ordre 0 et de degré 1 , c'est une dérivation de "Froelicher Nijenhuis" qui, à une 1cochaîne, fait correspondre sa différentielle de Koszul. Cet opérateur n'est donc rien 
d'autre que la différentielle de Koszul

$$
\delta: C^{p}(\mathfrak{g}, \mathbb{C}) \rightarrow C^{p+1}(\mathfrak{g}, \mathbb{C}) .
$$

Si bien, pour finir que :

$$
\begin{aligned}
T d_{p}\left(\boldsymbol{D} \otimes V_{p}\right) & \left.\left.=(-1)^{p}\left[\delta\left(V_{p}\right\rfloor \operatorname{vol}_{\mathfrak{g}}\right) \otimes \boldsymbol{D}^{\top+\triangle}+\sum_{k=1}^{k=n} e_{*}^{k} \wedge V_{p}\right\rfloor \operatorname{vol}_{\mathfrak{g}} \otimes e_{k} \boldsymbol{D}^{\top+\triangle}\right] \\
& =(-1)^{p} D^{1,0} T\left(\boldsymbol{D} \otimes V_{p}\right) .
\end{aligned}
$$

L'algèbre linéaire la plus élémentaire montrant que $T$ est un isomorphisme, ce résultat montre déjà que le complexe $\boldsymbol{C}^{\bullet}\left(\mathfrak{g},{ }^{g} \boldsymbol{U}\left(\mathfrak{g}, \mathcal{O}_{M}\right)\right)$ est exact sauf peut-être en "• $=n$ ". Ceci dit, soit maintenant l'augmentation :

$$
C^{n}\left(\mathfrak{g},{ }^{g} \boldsymbol{U}\left(\mathfrak{g}, \mathcal{O}_{M}\right)\right) \ni \operatorname{vol}_{\mathfrak{g}} \otimes D \stackrel{\varepsilon}{\mapsto} \operatorname{vol}_{\mathfrak{g}} \cdot \boldsymbol{D} \in C^{n}\left(\mathfrak{g}, \mathcal{O}_{M}\right),
$$

et toujours aussi l'augmentation $d_{0}: \boldsymbol{U}\left(\mathfrak{g}, \mathcal{O}_{M}\right) \rightarrow \mathcal{O}_{M}$ de noyau $\boldsymbol{I}\left(\mathfrak{g}, \mathcal{O}_{M}\right)$. Si on observe que, pour $\boldsymbol{D} \in \boldsymbol{I}\left(\mathfrak{g}, \mathcal{O}_{M}\right)^{\top+\triangle}$ on a $\operatorname{vol}_{\mathfrak{g}} \cdot \boldsymbol{D}=0$, et qu'enfin $\mathcal{O}_{M} \subset \boldsymbol{U}\left(\mathfrak{g}, \mathcal{O}_{M}\right)$ est laissé invariant par l'antiautomorphisme $T+\triangle$, on voit que :

$$
\varepsilon T(\boldsymbol{D})=d_{0}(\boldsymbol{D}) \operatorname{vol}_{\mathfrak{g}}
$$

ce qui achève de montrer que $\boldsymbol{C}^{\bullet}\left(\mathfrak{g},{ }^{g} \boldsymbol{U}\left(\mathfrak{g}, \mathcal{O}_{M}\right)\right)$ avec l'augmentation $\varepsilon$ est une résolution globale relativement "projective" du $\boldsymbol{U}\left(\mathfrak{g}, \mathcal{O}_{M}\right)$-module à droite $\boldsymbol{C}^{n}\left(\mathfrak{g}, \mathcal{O}_{M}\right)$ :

$$
\begin{gathered}
\cdots \stackrel{D^{1,0}}{\longrightarrow} \boldsymbol{C}^{p}\left(\mathfrak{g},{ }^{g} \boldsymbol{U}\left(\mathfrak{g}, \mathcal{O}_{M}\right)\right) \stackrel{D^{1,0}}{\longrightarrow} \boldsymbol{C}^{p+1}\left(\mathfrak{g},{ }^{g} \boldsymbol{U}\left(\mathfrak{g}, \mathcal{O}_{M}\right)\right) \stackrel{D^{1,0}}{\longrightarrow} \cdots \\
\stackrel{D^{1,0}}{\longrightarrow} \boldsymbol{C}^{n}\left(\mathfrak{g},{ }^{g} \boldsymbol{U}\left(\mathfrak{g}, \mathcal{O}_{M}\right)\right) \stackrel{\varepsilon}{\rightarrow} \boldsymbol{C}^{n}\left(\mathfrak{g}, \mathcal{O}_{M}\right) \rightarrow 0
\end{gathered}
$$

Observons que l'exactitude de ce complexe augmenté est plus surprenante que celle du complexe $\boldsymbol{\Omega}_{M}^{\bullet} \otimes_{\mathcal{O}_{M}} \mathcal{D}_{M}$, pour la raison que, si le complexe $\boldsymbol{\Omega}_{M}^{\bullet}$ avec la différentielle de de Rham est classiquement exact (sauf en zéro), il n'en est en général absolument pas de même du complexe $\boldsymbol{C}^{\bullet}\left(\mathfrak{g}, \mathcal{O}_{M}\right)$ des cochaînes totales holomorphes, i.e. encore les faisceaux de cohomologie totale $\mathcal{E}^{\bullet} \boldsymbol{U}_{\left(\mathfrak{g}, \mathcal{O}_{M}\right)}\left(\mathcal{O}_{M}, \mathcal{O}_{M}\right)$ peuvent fort bien être non nuls en dimension différente de zéro.

Le lecteur déduira aisément, de tout ce qui précède et si $\mathcal{F}$ est un $\boldsymbol{U}\left(\mathfrak{g}, \mathcal{O}_{M}\right)$-module à gauche, qu'alors le faisceau de cohomologie totale $\mathcal{E} x t_{\boldsymbol{U}\left(\mathfrak{g}, \mathcal{O}_{M}\right)}^{p}\left(\mathcal{O}_{M}, \mathcal{F}\right)$ est aussi le faisceau

$$
\mathcal{T} \operatorname{or}_{n-p}^{U\left(\mathfrak{g}, \mathcal{O}_{M}\right)}\left(\boldsymbol{C}^{n}\left(\mathfrak{g}, \mathcal{O}_{M}\right), \mathcal{F}\right) .
$$

Ce dernier résultat doit être vu comme la forme que prend, dans notre contexte particulier, le résultat de dualité homologique qu'on trouve dans [3] (corollaire 5.2.2) lui même généralisé par la notion de "duality Lie-Rinehart algebra" qui se trouve dans $[\mathbf{1 4}]$, sous la dénomination de "Poincaré duality".

On déduit aussi que, de même l'on peut interpréter "concrétement" le $0^{\text {eme }}$ faisceau de cohomologie totale $\mathcal{E} x t_{\boldsymbol{U}\left(\mathfrak{g}, \mathcal{O}_{M}\right)}^{0}\left(\mathcal{O}_{M}, \mathcal{F}\right)$ comme celui, désigné par $\mathcal{F}^{\mathfrak{g}}$ à la fin du 1.1., des germes de sections $\mathfrak{g}$-invariantes, on peut utiliser l'isomorphisme donné ici pour caractériser "concrétement" le $n^{e m e}$ faisceau de cohomologie totale 
$\mathcal{E} x t_{\boldsymbol{U}\left(\mathfrak{g}, \mathcal{O}_{M}\right)}^{n}\left(\mathcal{O}_{M}, \mathcal{F}\right)$, i.e. à tout le moins dans le cas unimodulaire $\triangle=0$, de voir que

$$
\mathcal{E} x t_{\boldsymbol{U}\left(\mathfrak{g}, \mathcal{O}_{M}\right)}^{n}\left(\mathcal{O}_{M}, \mathcal{F}\right)={ }^{d} \mathcal{O}_{M} \otimes_{\mathcal{O}_{M}} \mathcal{F}=\mathcal{F} / \mathfrak{g} \mathcal{F}
$$

où $\mathfrak{g} \mathcal{F}$ désigne les combinaisons linéaires sur $\mathbb{C}$ des germes de sections $\boldsymbol{\gamma}_{*}(\mathbf{u}) \sigma$ lorsque $\mathbf{u} \in \mathfrak{g}$.

\section{5. $\quad \boldsymbol{U}\left(\mathfrak{g}, \mathcal{O}_{M}\right)$-modules relativement injectifs.}

On définit classiquement les $\boldsymbol{U}\left(\mathfrak{g}, \mathcal{O}_{M}\right)$-modules injectifs $\mathcal{I}$ comme ceux pour lesquels l'application du foncteur contravariant $\operatorname{End}_{\boldsymbol{U}\left(\mathfrak{g}, \mathcal{O}_{M}\right)}(-, \mathcal{I})$ à une suite exacte courte :

$$
0 \rightarrow \mathcal{A}^{\prime} \rightarrow \mathcal{A} \rightarrow \mathcal{A}^{\prime \prime} \rightarrow 0
$$

de $\boldsymbol{U}\left(\mathfrak{g}, \mathcal{O}_{M}\right)$-modules permet d'obtenir une nouvelle suite exacte. Si maintenant $\mathcal{N}$ est un $\boldsymbol{U}\left(\mathfrak{g}, \mathcal{O}_{M}\right)$-module tel que l'application du foncteur $\operatorname{Hom}_{\boldsymbol{U}\left(\mathfrak{g}, \mathcal{O}_{M}\right)}(-, \mathcal{N})$ à toute suite exacte comme ci-dessus, qui soit de plus globalement scindée en tant que $\mathcal{O}_{M^{-}}$ module, redonne encore une suite exacte, on dira que $\mathcal{N}$ est relativement injectif. Avec cette définition, on voit que l'on est exactement dans le cadre de l'algèbre homologique relative de [12]. En considérant en effet la classe $\mathcal{M}^{0}$ des monomorphismes de $\boldsymbol{U}\left(\mathfrak{g}, \mathcal{O}_{M}\right)$-modules $0 \rightarrow \mathcal{A} \rightarrow \mathcal{B}$, tels que $\mathcal{A}$ soit un facteur direct de $\mathcal{B}$ en tant que $\mathcal{O}_{M}$-module, les $\boldsymbol{U}\left(\mathfrak{g}, \mathcal{O}_{M}\right)$-modules relativement injectifs ne sont autres que les objets $\mathcal{M}^{0}$-injectifs dans $\left(\boldsymbol{U}\left(\mathfrak{g}, \mathcal{O}_{M}\right)\right)$. La classe des $\mathcal{M}^{0}$-monomorphismes présente l'intérêt d'être fermée et, mieux encore, d'être injective. La démonstration en est "standard".

On indique alors un autre moyen, qui nous sera d'ailleurs fort utile, d'obtenir $\operatorname{des} \boldsymbol{U}\left(\mathfrak{g}, \mathcal{O}_{M}\right)$-modules $\mathcal{M}^{0}$-injectifs en observant qu'il est possible, en utilisant entre autres la formule d'adjonction "exotique", de montrer que si $\mathcal{I}$ est un $\boldsymbol{U}\left(\mathfrak{g}, \mathcal{O}_{M}\right)$ module injectif et $\mathcal{B}$ un $\boldsymbol{U}\left(\mathfrak{g}, \mathcal{O}_{M}\right)$-module alors, le $\boldsymbol{U}\left(\mathfrak{g}, \mathcal{O}_{M}\right)$-module ( $c f$. le 1er) du 2.3.) $\mathcal{H o m}_{\mathcal{O}_{M}}(\mathcal{B}, \mathcal{I})$ est un faisceau flasque qui est $\mathcal{M}^{0}$-injectif.

Indiquons aussi dans la catégorie $\operatorname{Mod}\left(\boldsymbol{U}\left(\mathfrak{g}, \mathcal{O}_{M}\right)\right)$ une classe injective de monomorphismes qui contient tous ceux qui sont localement $\mathcal{O}_{M}$-scindés. Pour cela nous allons faire un détour par la catégorie $\operatorname{Mod}\left(\boldsymbol{U}\left(\mathfrak{g}, \mathcal{C}_{M}^{\infty}\right)\right)$. Désignons, dans cette catégorie $\operatorname{Mod}\left(\boldsymbol{U}\left(\mathfrak{g}, \mathcal{C}_{M}^{\infty}\right)\right)$, par $\boldsymbol{\mu}^{\infty}$ la classe des monomorphismes $\mathcal{C}_{M}^{\infty}$-scindés. On voit immédiatement que cette classe est conservée par la composition. Il est alors encore aisé de vérifier que $\boldsymbol{\mu}^{\infty}$ est une classe fermée et injective de monomorphismes.

On va maintenant considérer ( $c f$. le 6) 2.3.) le foncteur

$$
\mathrm{L}: \operatorname{Mod}\left(\boldsymbol{U}\left(\mathfrak{g}, \mathcal{O}_{M}\right)\right) \rightarrow \operatorname{Mod}\left(\boldsymbol{U}\left(\mathfrak{g}, \mathcal{C}_{M}^{\infty}\right)\right)
$$

dît de "lissification" et donné par

$$
\mathrm{L}(\mathcal{F})=C_{M}^{\infty} \otimes^{g} \mathcal{O}_{M} \mathcal{F}\left(=U\left(\mathfrak{g}, \mathcal{C}_{M}^{\infty}\right) \otimes_{\boldsymbol{U}\left(\mathfrak{g}, \mathcal{O}_{M}\right)} \mathcal{F}\right) .
$$

Soit maintenant $\alpha: \mathcal{F} \rightarrow \mathcal{F}^{\prime}$ un morphisme dans $\operatorname{Mod}\left(\boldsymbol{U}\left(\mathfrak{g}, \mathcal{O}_{M}\right)\right)$; alors $\operatorname{L} \alpha: \mathrm{L}(\mathcal{F}) \rightarrow$ $\mathrm{L}\left(\mathcal{F}^{\prime}\right)$ est un morphisme dans $\operatorname{Mod}\left(\boldsymbol{U}\left(\mathfrak{g}, \mathcal{C}_{M}^{\infty}\right)\right)$. La platitude du $\mathcal{O}_{M}$-module $\mathcal{C}_{M}^{\infty}$ implique, si $\alpha$ est un monomorphisme, qu'alors $\mathrm{L}(\alpha)$ est lui aussi un monomorphisme. Mais en fait $\mathcal{C}_{M}^{\infty}$ est même fidèlement plat (résultat bien connu dû à Malgrange) ce qui réciproquement montre, si $\mathrm{L}(\alpha)$ est un monomorphisme, qu'alors $\alpha$ en est un lui aussi. Enfin, si U est le foncteur d'oubli comme dans le 6) de 2.3 et que $\mathcal{F}$ est un $\boldsymbol{U}\left(\mathfrak{g}, \mathcal{O}_{M}\right)$-module, l'adjonction de l'identité $\mathrm{L} \mathcal{F} \rightarrow \mathrm{L} \mathcal{F}$ définit un morphisme de 
$\boldsymbol{U}\left(\mathfrak{g}, \mathcal{O}_{M}\right)$-modules $\delta: \mathcal{F} \rightarrow$ UL $\mathcal{F}$ et la fidèle platitude permet de voir que cette flèche est un monomorphisme.

On désigne par $\mathcal{M}^{\infty}$ la classe des morphismes de $\operatorname{Mod}\left(\boldsymbol{U}\left(\mathfrak{g}, \mathcal{O}_{M}\right)\right)$ tels que $\mathrm{L}(\alpha)$ soit dans la classe $\boldsymbol{\mu}^{\infty}$. La fidèle platitude permet de voir que $\mathcal{M}^{\infty}$ est une classe de monomorphismes qui permet de dire ce qu'est un objet $\mathcal{M}^{\infty}$-injectif. En désignant par $\mathcal{M}^{U}$ la classe (injective) de tous les monomorphismes de la catégorie $\operatorname{Mod}\left(\boldsymbol{U}\left(\mathfrak{g}, \mathcal{O}_{M}\right)\right)$, on peut montrer :

Proposition 3.6. La classe $\mathcal{M}^{\infty}$ est une classe injective de monomorphismes dans la catégorie abélienne $\operatorname{Mod}\left(\boldsymbol{U}\left(\mathfrak{g}, \mathcal{O}_{M}\right)\right.$ ). (Rappelons que nous avons vu plus haut que $\mathcal{M}^{0}$ était une classe injective.) On a donc la suite croissante (par "inclusion") de classes injectives de $\boldsymbol{U}\left(\mathfrak{g}, \mathcal{O}_{M}\right)$-monomorphismes : $\mathcal{M}^{0} \subset \mathcal{M}^{\infty} \subset \mathcal{M}^{U}$.

\subsection{Un résultat de finitude.}

Dans [19] on signale dans la note 20 au bas de la page 129, l'existence probable d'un objet cohomologique comme aussi, l'existence de deux suites spectrales que nous précisons qui approchent cet objet. Les notions introduites ici, comme aussi l'utilisation des $\boldsymbol{U}\left(\mathfrak{g}, \mathcal{O}_{M}\right)$-modules relativement injectifs, vont nous permettre d'éclaircir le propos de cette note en bas de page. Tout d'abord, si $\mathcal{F}$ est un $\boldsymbol{U}\left(\mathfrak{g}, \mathcal{O}_{M}\right)$-module, nous dirons que $\mathcal{F}$ est acyclique pour la cohomologie totale ssi les groupes de cohomologie totale $\mathbf{H}^{i}(\mathfrak{g}, \mathcal{M})$ sont nuls pour tout $i>0$. Le contexte étant clair on dira plus brièvement que $\mathcal{F}$ est $t$ t-acyclique. Sans développer les démonstrations, énonçons :

Proposition 3.7. Soit $\mathcal{F}$ un $\boldsymbol{U}\left(\mathfrak{g}, \mathcal{O}_{M}\right)$-module $\mathcal{M}^{0}$-injectif.

(i) Le $i^{\text {eme }}$ faisceau de cohomologie totale $\mathcal{E} x t_{\boldsymbol{U}\left(\mathfrak{g}, \mathcal{O}_{M}\right)}^{i}\left(\mathcal{O}_{M}, \mathcal{F}\right)=0$ s'annule pour tout $i>0$, et donc : $\mathbf{H}^{i}(\mathfrak{g}, \mathcal{F})=H^{i}\left(M, \mathcal{F}^{\mathfrak{g}}\right)$ pour tout $i \geqslant 0$.

(ii) En particulier, si on sait de plus que $\mathcal{F}^{\mathfrak{g}}$ est flasque, alors $\mathcal{F}$ est tt-acyclique.

(iii) Si $\mathcal{I}$ est un $\boldsymbol{U}\left(\mathfrak{g}, \mathcal{O}_{M}\right)$-module injectif et que $\mathcal{B}$ est un $\boldsymbol{U}\left(\mathfrak{g}, \mathcal{O}_{M}\right)$-module quelconque, le $\boldsymbol{U}\left(\mathfrak{g}, \mathcal{O}_{M}\right)$-module $\mathcal{H o m}_{\mathcal{O}_{M}}(\mathcal{B}, \mathcal{I})$ est tt-acyclique.

Démonstration. Le scindage relatif en $p>0$ du complexe $\mathcal{L}_{\bullet}$ défini plus haut montre, si $\mathcal{F}$ est relativement injectif, que la cohomologie faisceautique du complexe

$$
\mathcal{H o m}_{\boldsymbol{U}\left(\mathfrak{g}, \mathcal{O}_{M}\right)}\left(\mathcal{L}_{\bullet}, \mathcal{F}\right)
$$

est nulle en $p>0$ et vaut $\mathcal{F}^{\mathfrak{g}}$ en $p=0$. L' hypercohomologie de ce complexe n'est donc rien d'autre que la cohomologie usuelle du faisceau $\mathcal{F}^{\mathfrak{g}}$, mais, comme nous l'avons vu au 3.1., cette hypercohomologie est aussi la cohomologie totale de $\mathcal{F}$, ce qui achève de montrer (i). (ii) en résulte alors immédiatement. Enfin (voir sous-section 3.5.), le $\boldsymbol{U}\left(\mathfrak{g}, \mathcal{O}_{M}\right)$-module $\mathcal{H o m}_{\mathcal{O}_{M}}(\mathcal{B}, \mathcal{I})$ est relativement injectif et

$$
\mathcal{H o m}_{\mathcal{O}_{M}}(\mathcal{B}, \mathcal{I})^{\mathfrak{g}}=\mathcal{H o m}_{\boldsymbol{U}\left(\mathfrak{g}, \mathcal{O}_{M}\right)}(\mathcal{B}, \mathcal{I})
$$

est flasque par le 7.3.2. de [10]; si bien que le (iii) résulte immédiatement de (ii).

On en déduit :

Proposition 3.8. Soient $\mathcal{F}$ et $\mathcal{G}$ deux $\boldsymbol{U}\left(\mathfrak{g}, \mathcal{O}_{M}\right)$-modules à gauche. Alors il existe une suite spectrale de deuxième terme la cohomologie totale $\mathbf{H}^{p}\left(\mathfrak{g}, \mathcal{E} x t_{\mathcal{O}_{M}}^{q}(\mathcal{F}, \mathcal{G})\right)$ et qui aboutit vers $\operatorname{Ext}_{\boldsymbol{U}\left(\mathfrak{g}, \mathcal{O}_{M}\right)}^{p+q}(M ; \mathcal{F}, \mathcal{G})$. 
Démonstration. Brièvement, comme nous avons montré plus haut que le premier foncteur $\mathcal{H o m}_{\mathcal{O}_{M}}(\mathcal{F},-)$ transformait tout $\boldsymbol{U}\left(\mathfrak{g}, \mathcal{O}_{M}\right)$-module injectif en un objet "ttacyclique", le résultat se déduit donc du théorème de composition des foncteurs de Grothendieck, étant entendu que $\mathcal{E} x t_{\mathcal{O}_{M}}^{q}(\mathcal{F},-)$ est le $q^{\text {eme }}$ foncteur dérivé du premier foncteur, et $\mathbf{H}^{p}(\mathfrak{g},-)$ le $p^{\text {eme }}$ du second.

Utilisons alors le (ii) de la proposition 3.5 pour en donner une généralisation :

Proposition 3.9. Soient $\mathcal{F}$ et $\mathcal{G}$ deux $\boldsymbol{U}\left(\mathfrak{g}, \mathcal{O}_{M}\right)$-modules à gauche. Alors il existe une suite spectrale de deuxième terme la cohomologie de Chevalley-Eilenberg

$$
H^{p}\left(\mathfrak{g}, \operatorname{Ext}_{\mathcal{O}_{M}}^{q}(M ; \mathcal{F}, \mathcal{G})\right)
$$

dans le $\mathfrak{g}$-module $\operatorname{Ext}_{\mathcal{O}_{M}}^{q}(M ; \mathcal{F}, \mathcal{G})$ et d'aboutissement

$$
\operatorname{Ext}_{\boldsymbol{U}\left(\mathfrak{g}, \mathcal{O}_{M}\right)}^{p+q}(M ; \mathcal{F}, \mathcal{G})
$$

Démonstration. Via le théorème de composition de Grothendieck, on voit que la proposition résulte essentiellement de ce que, si $\mathcal{G}$ un $\boldsymbol{U}\left(\mathfrak{g}, \mathcal{O}_{M}\right)$-module injectif, alors le $\mathfrak{g}$-module $\operatorname{Hom}_{\mathcal{O}_{M}}(M ; \mathcal{F}, \mathcal{G})$ est acyclique pour la cohomologie d'algèbre de Lie complexe; i.e.

$$
H^{p}\left(\mathfrak{g}, \operatorname{Hom}_{\mathcal{O}_{M}}(M ; \mathcal{F}, \mathcal{G})\right)=0
$$

pour $p>0$. On a vu, en effet, que le $\boldsymbol{U}\left(\mathfrak{g}, \mathcal{O}_{M}\right)$-module $\mathcal{H}_{\text {om }}{\mathcal{O}_{M}}(\mathcal{F}, \mathcal{G})$ est flasque et que sa cohomologie de faisceau est nulle en dimension $>0$. En particulier le (ii) de la proposition 3.5 s'applique pour dire que la cohomologie totale de ce $\boldsymbol{U}\left(\mathfrak{g}, \mathcal{O}_{M}\right)$ module est :

$$
\mathbf{H}^{p}\left(\mathfrak{g}, \mathcal{H o m}_{\mathcal{O}_{M}}(\mathcal{F}, \mathcal{G})\right)=H^{p}\left(\mathfrak{g}, \operatorname{Hom}_{\mathcal{O}_{M}}(M ; \mathcal{F}, \mathcal{G})\right)
$$

Mais, la proposition 3.7(iii) affirme aussi que le $\boldsymbol{U}\left(\mathfrak{g}, \mathcal{O}_{M}\right)$-module $\mathcal{H}_{o m_{\mathcal{O}_{M}}}(\mathcal{F}, \mathcal{G})$ est tt-acyclique. La proposition se déduit donc de l'égalité écrite à l'instant.

On complétera ce 3.6. par l'observation qu'un objet $\mathcal{M}^{0}$-injectif peut avoir une cohomologie totale non nulle en dimension strictement positive, et qu'en conséquence la flèche naturelle de la cohomologie totale $\mathcal{M}^{0}$-relative $\operatorname{Ext}_{\mathcal{M}^{0}}\left(M ; \mathcal{O}_{M}, \mathcal{F}\right)$ vers la cohomologie totale "absolue"

$$
\operatorname{Ext}_{\boldsymbol{U}\left(\mathfrak{g}, \mathcal{O}_{M}\right)}^{i}\left(M ; \mathcal{O}_{M}, \mathcal{F}\right)
$$

n'est pas, en général un isomorphisme. Par contre, au moyen du (i) de la proposition du début du présent 3.6., on peut voir que la flèche du faisceau de cohomologie totale $\mathcal{M}^{0}$-relative vers le faisceau de cohomologie totale "absolue" est un isomorphisme :

$$
\mathcal{E} x t_{\mathcal{M}^{0}}^{i}\left(\mathcal{O}_{M}, \mathcal{F}\right) \approx \mathcal{E} x t_{\boldsymbol{U}\left(\mathfrak{g}, \mathcal{O}_{M}\right)}^{i}\left(\mathcal{O}_{M}, \mathcal{F}\right)
$$

\section{Suite spectrale d'ancrage.}

\section{1. $U\left(\mathfrak{g}, \mathcal{O}_{M}\right)$-modules avec $(1,0)$-dérivations covariantes, au sens large, le long des orbites.}

$\mathrm{Au} c$ ) de 2.2., on a considéré des faisceaux $\mathcal{F}$ munis d'une $(1,0)$-connexion "le long des orbites". Pour chaque section locale $m$ d'un tel faisceau, on désigne par ${ }^{e v} \nabla m$, 
l'image de $\nabla^{\mathfrak{g}} m$ par la flèche

$$
{ }^{e v} \boldsymbol{\Omega}_{M}^{1} \otimes \mathcal{F} \rightarrow C^{1}(\mathfrak{g}, \mathcal{F}) .
$$

Par la formule $D^{1,0} m={ }^{e v} \nabla m$ on définit sur $\mathcal{F}$ a une structure de $\boldsymbol{U}\left(\mathfrak{g}, \mathcal{O}_{M}\right)$-module par (notation de l'annexe 5) l'identité

$$
\left.\left.\boldsymbol{\gamma}_{*}(\mathbf{u}) m=\mathbf{u}\right\rfloor D^{1,0} m=\mathbf{u}_{M}\right\rfloor \nabla^{\mathfrak{g}} m
$$

(l'écriture $\mathbf{u}\rfloor \nabla^{\mathfrak{g}} m$ aurait aussi un sens). En fait, en désignant par $\mathcal{K}$ le faisceau de Killing comme au (a) de 2.1., la surjection de $\mathcal{O}_{M}$-modules, $\boldsymbol{E}_{1}\left(\mathfrak{g}, \mathcal{O}_{M}\right) \rightarrow \mathcal{K}$, montre l'inclusion :

$$
\mathcal{H o m}_{\mathcal{O}_{M}}(\mathcal{K}, \mathcal{F}) \hookrightarrow \mathcal{H o m}_{\mathcal{O}_{M}}\left(\boldsymbol{E}_{1}\left(\mathfrak{g}, \mathcal{O}_{M}\right), \mathcal{F}\right)=C^{1}(\mathfrak{g}, \mathcal{F}) .
$$

Avec les hypothèses faites ici sur $\mathcal{F}$, il s'avère qu'en fait $D^{1,0} m$ est une section locale de $\mathcal{H} m_{\mathcal{O}_{M}}(\mathcal{K}, \mathcal{F})$, ce qui amène naturellement à la généralisation :

Définition 4.1. On dit que la structure de $\boldsymbol{U}\left(\mathfrak{g}, \mathcal{O}_{M}\right)$-module provient d'une $(1,0)$ dérivation covariante au sens large le long des orbites ssi la fléche

$$
D^{1,0}: \mathcal{F} \rightarrow C^{1}(\mathfrak{g}, \mathcal{F})
$$

se factorise via

$$
\mathcal{F} \rightarrow \mathcal{H o m}_{\mathcal{O}_{M}}(\mathcal{K}, \mathcal{F}) \hookrightarrow C^{1}(\mathfrak{g}, \mathcal{F}) .
$$

Afin de reformuler cette définition, commençons ( $c f . \quad$ c) du 2.3) par poser :

$$
\check{\mathcal{Q}} \stackrel{\text { def }}{=} \mathcal{H o m}_{\mathcal{O}_{M}}\left(\mathcal{Q}, \mathcal{O}_{M}\right) \text {. }
$$

Evidemment :

$$
\check{\mathcal{Q}}=\operatorname{ker}\left(\boldsymbol{E}_{1}\left(\mathfrak{g}, \mathcal{O}_{M}\right) \rightarrow \boldsymbol{T}^{1,0} M\right) .
$$

Introduisons alors l'idéal bilatère faisceautique

$$
(\check{\mathcal{Q}})=\boldsymbol{U}\left(\mathfrak{g}, \mathcal{O}_{M}\right) \check{\mathcal{Q}} \boldsymbol{U}\left(\mathfrak{g}, \mathcal{O}_{M}\right)
$$

engendré par $\check{\mathcal{Q}} \subset \boldsymbol{U}\left(\mathfrak{g}, \mathcal{O}_{M}\right)$. Sur un "ouvert de Zariski" non vide, i.e. sur le complémentaire d'un sous-ensemble analytique strict fermé, cet idéal est précisément le noyau :

$$
\operatorname{ker}\left[\boldsymbol{U}\left(\mathfrak{g}, \mathcal{O}_{M}\right) \rightarrow \mathcal{D}_{M}\right]
$$

Via la flèche surjective :

$$
\boldsymbol{U}\left(\mathfrak{g}, \mathcal{O}_{M}\right) \rightarrow \boldsymbol{U}\left(\mathfrak{g}, \mathcal{O}_{M}\right) /(\check{\mathcal{Q}}),
$$

tout $\boldsymbol{U}\left(\mathfrak{g}, \mathcal{O}_{M}\right) /(\check{\mathcal{Q}})$-module est un $\boldsymbol{U}\left(\mathfrak{g}, \mathcal{O}_{M}\right)$-module. La sous-catégorie pleine

$$
\operatorname{Mod}\left(\boldsymbol{U}\left(\mathfrak{g}, \mathcal{O}_{M}\right) /(\check{\mathcal{Q}})\right)
$$

de $\operatorname{Mod}\left(\boldsymbol{U}\left(\mathfrak{g}, \mathcal{O}_{M}\right)\right)$ est la sous-catégorie abélienne des modules qui contiennent $\breve{\mathcal{Q}}$ dans leur annulateur.

La suite exacte :

$$
0 \rightarrow \check{\mathcal{Q}} \rightarrow \boldsymbol{E}_{1}\left(\mathfrak{g}, \mathcal{O}_{M}\right) \rightarrow \mathcal{K} \rightarrow 0
$$

permet donc d'affirmer : 
Observation 4.2. La sous-catégorie $\operatorname{Mod}\left(\boldsymbol{U}\left(\mathfrak{g}, \mathcal{O}_{M}\right) /(\check{\mathcal{Q}})\right)$ n'est rien d'autre que celle des $\boldsymbol{U}\left(\mathfrak{g}, \mathcal{O}_{M}\right)$-modules avec une dérivation covariante au sens large le long des orbites.

On fera encore une toute dernière observation : Le sous-anneau

$$
\mathcal{O}_{M} \subset \boldsymbol{U}\left(\mathfrak{g}, \mathcal{O}_{M}\right) /(\check{\mathcal{Q}})
$$

est évidemment un $\boldsymbol{U}\left(\mathfrak{g}, \mathcal{O}_{M}\right) /(\check{\mathcal{Q}})$-module et si $\boldsymbol{X}$ est une section locale de $\mathcal{K} \subset$ $\boldsymbol{U}\left(\mathfrak{g}, \mathcal{O}_{M}\right) /(\check{\mathcal{Q}})$, on a dans cet anneau la formule de commutation $\boldsymbol{x} f=f \boldsymbol{X}+\boldsymbol{X} \cdot f$, où $\boldsymbol{X} \cdot f=\mathcal{L}_{\boldsymbol{X}_{M}} f$. Observons, par contre, que le $\boldsymbol{U}\left(\mathfrak{g}, \mathcal{O}_{M}\right)$-module $\boldsymbol{\Omega}_{M}^{p}$ n'est pas un $\boldsymbol{U}\left(\mathfrak{g}, \mathcal{O}_{M}\right) /(\check{\mathcal{Q}})$-module pour $1 \leqslant p \leqslant m=\operatorname{dim}_{\mathbb{C}} M$.

Si $\mathcal{F}$ est un $\boldsymbol{U}\left(\mathfrak{g}, \mathcal{O}_{M}\right)$-module, on désigne indifféremment par $\mathcal{F}^{\check{\mathcal{Q}}}$ ou $\mathcal{F}_{\boldsymbol{\gamma}_{*}(\breve{\mathcal{Q}})=0}$, le faisceau des germes de section $\sigma$ de $\mathcal{F}$ pour lesquelles $\boldsymbol{\gamma}_{*}(\boldsymbol{X}) \sigma=0$, dès que $\boldsymbol{X}$ est une section de $\check{\mathcal{Q}} \subset \boldsymbol{E}_{1}\left(\mathfrak{g}, \mathcal{O}_{M}\right)$. On énonce alors :

Proposition 4.3. Le foncteur $\mathcal{F} \mapsto \mathcal{F}^{\mathcal{Q}}$ possède les propriétés suivantes :

(i) C'est un foncteur additif de la catégorie abélienne $\operatorname{Mod}\left(\boldsymbol{U}\left(\mathfrak{g}, \mathcal{O}_{M}\right)\right)$ dans la sous-catégorie $\operatorname{Mod} \boldsymbol{U}\left(\mathfrak{g}, \mathcal{O}_{M}\right) /(\check{\mathcal{Q}})$ des modules avec une dérivation au sens large le long des orbites, qui est exact à gauche.

(ii) Si $\mathcal{I}$ est un $\boldsymbol{U}\left(\mathfrak{g}, \mathcal{O}_{M}\right)$-module injectif, alors $\mathcal{I}^{\check{\mathcal{Q}}}$ est un $U\left(\mathfrak{g}, \mathcal{O}_{M}\right) /(\check{\mathcal{Q}})$-module injectif.

Démonstration. Démonstration de (i) : La $\mathfrak{g}$-invariance de $\check{\mathcal{Q}} \subset \boldsymbol{T}^{1,0}$ implique celle de $\mathcal{F}^{\breve{Q}}$, qui est donc un sous- $\boldsymbol{U}\left(\mathfrak{g}, \mathcal{O}_{M}\right)$-module de $\mathcal{F}$. Si

$$
0 \rightarrow \mathcal{F}^{\prime} \rightarrow \mathcal{F} \rightarrow \mathcal{F}^{\prime \prime} \rightarrow 0
$$

est une suite exacte de $\boldsymbol{U}\left(\mathfrak{g}, \mathcal{O}_{M}\right)$-modules, l'injectivité de la flèche

$$
0 \rightarrow \operatorname{Hom}_{\boldsymbol{U}\left(\mathfrak{g}, \mathcal{O}_{M}\right)}\left(\boldsymbol{U}\left(\mathfrak{g}, \mathcal{O}_{M}\right) /(\check{\mathcal{Q}}), \mathcal{F}^{\prime}\right) \rightarrow \boldsymbol{U}\left(\mathfrak{g}, \mathcal{O}_{M}\right)\left(\boldsymbol{U}\left(\mathfrak{g}, \mathcal{O}_{M}\right) /(\check{\mathcal{Q}}), \mathcal{F}\right)
$$

montre l'exactitude recherchée. L'annulation de la composition :

$$
\mathcal{F}^{\check{\mathcal{Q}}} \stackrel{D^{1,0}}{\rightarrow} C^{1}(\mathfrak{g}, \mathcal{F}) \rightarrow \operatorname{Hom}_{\mathcal{O}_{M}}(\check{\mathcal{Q}}, \mathcal{F})
$$

montre que la flèche $D^{1,0}$ est à valeurs dans $\operatorname{Hom}_{\mathcal{O}_{M}}(\mathcal{K}, \mathcal{F})$, ce qui permet de conclure.

Pour montrer (ii), considérons un $\boldsymbol{U}\left(\mathfrak{g}, \mathcal{O}_{M}\right)$-module injectif $\mathcal{I}$ ainsi qu'un monomorphisme $\mathcal{A} \rightarrow \mathcal{B}$ de $\boldsymbol{U}\left(\mathfrak{g}, \mathcal{O}_{M}\right) /(\check{\mathcal{Q}})$-modules. Si $\phi: \mathcal{A} \rightarrow \mathcal{I}^{\check{\mathcal{Q}}}$ est un morphisme de $\boldsymbol{U}\left(\mathfrak{g}, \mathcal{O}_{M}\right) /(\check{\mathcal{Q}})$-modules, sa composée avec $\mathcal{I}^{\check{\mathcal{Q}}} \hookrightarrow \mathcal{I}$ s'étend en une flèche $\boldsymbol{U}\left(\mathfrak{g}, \mathcal{O}_{M}\right)$ linéaire $\mathcal{B} \rightarrow \mathcal{I}$. Mais alors, si $\boldsymbol{x}$ est une section locale de $\check{\mathcal{Q}}$, la $\boldsymbol{U}\left(\mathfrak{g}, \mathcal{O}_{M}\right)$-linéarité de cette flèche combinée avec la trivialité de l'action de $\boldsymbol{\gamma}_{*}(\boldsymbol{x})$ sur une section de $\mathcal{B}$, montre que cette flèche se factorise par une flèche $: \mathcal{B} \rightarrow \mathcal{I}^{\mathscr{\mathcal { Q }}}$ qui est évidemment l'élargissement $\boldsymbol{U}\left(\mathfrak{g}, \mathcal{O}_{M}\right) /(\check{\mathcal{Q}})$-linéaire recherché de $\phi$ à $\mathcal{B}$ tout entier. Ce qui achève de montrer le (ii).

Si maintenant $\mathcal{F}$ est un $\boldsymbol{U}\left(\mathfrak{g}, \mathcal{O}_{M}\right)$-module, il est immédiat de voir que le sousfaisceau $\mathcal{H o m}_{\boldsymbol{U}\left(\mathfrak{g}, \mathcal{O}_{M}\right) /(\check{\mathcal{Q}})}\left(\mathcal{O}_{M}, \mathcal{F}^{\check{\mathcal{Q}}}\right)$ des germes de $\mathcal{F}^{\check{\mathcal{Q}}}$ qui sont $\mathcal{K}$-invariants, n'est rien d'autre que le sous-faisceau $\mathcal{H}_{D^{1,0}}^{0}(\mathcal{F})$ des germes $\mathfrak{g}$-invariants. Ainsi donc 
le foncteur exact à gauche

$$
\mathcal{F} \mapsto \mathcal{H o m}_{\boldsymbol{U}\left(\mathfrak{g}, \mathcal{O}_{M}\right)}\left(\mathcal{O}_{M}, \mathcal{F}\right)
$$

de $\operatorname{Mod}\left(\boldsymbol{U}\left(\mathfrak{g}, \mathcal{O}_{M}\right)\right)$ vers la catégorie des faisceaux de $\mathbb{C}$-espaces vectoriels (et même à vrai dire dans la sous-catégorie des $\mathcal{O}_{M}^{\mathfrak{g}}$-modules) est-t-il le composé d'un premier foncteur exact à gauche :

$$
\mathcal{F} \mapsto \mathcal{H}_{\check{\mathcal{Q}}}^{0}(\mathcal{F}) \stackrel{\text { def }}{=} \mathcal{F}^{\check{\mathcal{Q}}}
$$

à valeurs dans $\operatorname{Mod}\left(\boldsymbol{U}\left(\mathfrak{g}, \mathcal{O}_{M}\right) /(\check{\mathcal{Q}})\right)$, avec $\mathcal{H o m}_{\boldsymbol{U}\left(\mathfrak{g}, \mathcal{O}_{M}\right) /(\check{\mathcal{Q}})}\left(\mathcal{O}_{M},-\right)$ lui aussi exact à gauche. La proposition qui précède montre que le premier foncteur envoie injectifs sur injectifs; si bien que l'on est exactement dans le cadre d'application du théorème de Grothendieck sur le foncteur dérivé d'un foncteur composé. Plus précisément, en désignant par

$$
\mathcal{H}_{\tilde{\mathcal{Q}}}^{q}(-)=\mathcal{E} x t_{\boldsymbol{U}\left(\mathfrak{g}, \mathcal{O}_{M}\right)}^{q}\left[\boldsymbol{U}\left(\mathfrak{g}, \mathcal{O}_{M}\right) /(\check{\mathcal{Q}}),-\right]
$$

le $q^{\text {eme }}$ foncteur dérivé à droite du premier foncteur, on voit immédiatement que :

Proposition 4.4. Soit $\mathcal{F}$ est un $\boldsymbol{U}\left(\mathfrak{g}, \mathcal{O}_{M}\right)$-module.

(i) Il existe une suite spectrale faisceautique de deuxième terme

$$
E_{2}^{p, q}=\mathcal{E} x t_{\boldsymbol{U}\left(\mathfrak{g}, \mathcal{O}_{M}\right) /(\check{\mathcal{Q}})}^{p}\left(\mathcal{O}_{M}, \mathcal{H}_{\check{\mathcal{Q}}}^{q}(\mathcal{F})\right)
$$

qui converge vers $\mathcal{E} x t_{\boldsymbol{U}\left(\mathfrak{g}, \mathcal{O}_{M}\right)}^{p+q}\left(\mathcal{O}_{M}, \mathcal{F}\right)$.

(ii) Il existe une suite spectrale de $\mathbb{C}$-espaces vectoriels de deuxième terme

$$
E_{2}^{p, q}=\operatorname{Ext}_{\boldsymbol{U}\left(\mathfrak{g}, \mathcal{O}_{M}\right) /(\check{\mathcal{Q}})}^{p}\left(M ; \mathcal{O}_{M}, \mathcal{H}_{\mathcal{Q}^{q}}^{q}(\mathcal{F})\right)
$$

qui converge vers $\operatorname{Ext}_{\boldsymbol{U}\left(\mathfrak{g}, \mathcal{O}_{M}\right)}^{p+q}\left(M ; \mathcal{O}_{M}, \mathcal{F}\right)$.

\subsection{Description "géométrique" d'un exemple.}

Soient $M$ une variété analytique complexe, $G$ un groupe de Lie complexe qui y agit holomorphiquement à gauche, et enfin $\mathcal{F}$ un faisceau analytique cohérent sur M ainsi qu'une $G$-action holomorphe équivariante sur $\mathcal{F}$. Si $\boldsymbol{m} \in M$, on désigne respectivement par $\operatorname{Stab}_{G}(\boldsymbol{m})$ et $\operatorname{Stab}_{G}^{0}(\boldsymbol{m})$, le stabilisateur de $\boldsymbol{m}$ dans $G$ et la composante connexe dudit stabilisateur dont $\mathfrak{s t a b}_{\mathfrak{g}}(\boldsymbol{m}) \subset \mathfrak{g}$ est l'algèbre de Lie commune. Ce sont deux sous-groupes fermés de $G$. On a évidemment la représentation linéaire naturelle de ces sous-groupes, comme aussi de leur algèbre de Lie $\mathfrak{s t a b}_{\mathfrak{g}}(\boldsymbol{m})$ dans la fibre géométrique $\mathcal{F}_{\boldsymbol{m}} / \mathfrak{m}_{\boldsymbol{m}} \mathcal{F}_{\boldsymbol{m}}$ (on désigne ici par $\mathfrak{m}_{\boldsymbol{m}} \subset \mathcal{O}_{M, \boldsymbol{m}}$, l'idéal des germes de fonctions holomorphes qui prennent la valeur zéro en $\boldsymbol{m}$ ) qui permet de considérer la cohomologie de Chevalley-Eilenberg

$$
H^{q}\left(\mathfrak{s t a b} \mathfrak{b}_{\mathfrak{g}}(\boldsymbol{m}), \mathcal{F}_{\boldsymbol{m}} / \mathfrak{m}_{\boldsymbol{m}} \mathcal{F}_{\boldsymbol{m}}\right) .
$$

Pour visualiser un tant soit peu cette suite spectrale, on se placera dans le contexte où $\mathcal{F}$ est un fibré vectoriel holomorphe de rang fini et où (ce qui est d'ailleurs alors génériquement vrai) $\mathcal{H}_{\check{\mathcal{Q}}}^{q}(\mathcal{F})$ est lui aussi un fibré vectoriel holomorphe (équivariant !) avec

$$
H^{q}\left(\mathfrak{s t a b} \mathfrak{b}_{\mathfrak{g}}(\boldsymbol{m}), \mathcal{F}_{\boldsymbol{m}} / \mathfrak{m}_{\boldsymbol{m}} \mathcal{F}_{\boldsymbol{m}}\right)
$$

comme fibre géométrique en un point $\boldsymbol{m} \in M$. 
Dans ce contexte particulier, et si $\boldsymbol{m} \in M$, considérons maintenant la restriction de ce fibré $\mathcal{H}_{\mathcal{Q}}^{q}(\mathcal{F})$ sur l'orbite $G \boldsymbol{m}$ immergée dans $M$. C'est un espace homogène complexe sous $G$ sur la restriction duquel $\mathcal{H}_{\mathcal{Q}}^{q}(\mathcal{F})$ est un fibré équivariant qui est donc complétement déterminé par la représentation linéaire qu'on peut en déduire :

$$
\varrho: \operatorname{Stab}_{G}(\boldsymbol{m}) \rightarrow H^{q}\left(\mathfrak{s t a b}(\boldsymbol{m}), \mathcal{F}_{\boldsymbol{m}} / \mathfrak{m}_{\boldsymbol{m}} \mathcal{F}_{\boldsymbol{m}}\right) .
$$

La chose essentielle ici est que cette représentation sur les cochaînes devient triviale au niveau cohomologique lorsque restreinte à la composante neutre $\operatorname{Stab}_{G}^{0}(\boldsymbol{m})$. Par ailleurs l'image réciproque sur $G \boldsymbol{m} \hookrightarrow M$ du fibré $\mathcal{H}_{\tilde{Q}}^{q}(\mathcal{F})$ n'est rien d'autre (cf. [6], [19] et [21]) que le fibré équivariant

$$
G \times{ }^{\varrho} H^{q}\left(\mathfrak{s t a b}(\boldsymbol{m}), \mathcal{F}_{\boldsymbol{m}} / \mathfrak{m}_{\boldsymbol{m}} \mathcal{F}_{\boldsymbol{m}}\right),
$$

de fibre type la cohomologie de Chevalley-Eilenberg. Mais comme, par ailleurs,

$$
G \times{ }^{\varrho} H^{q}\left(\mathfrak{s t a b}(\boldsymbol{m}), \mathcal{F}_{\boldsymbol{m}} / \mathfrak{m}_{\boldsymbol{m}} \mathcal{F}_{\boldsymbol{m}}\right)
$$

est en fait la restriction de $\mathcal{H}_{\tilde{\mathcal{Q}}}^{q}(\mathcal{F})$ à $G \boldsymbol{m}$, la factorisation possible de $\varrho$ en une représentation du groupe discret $\operatorname{Stab}_{G}(\boldsymbol{m}) / \operatorname{Stab}_{G}^{0}(\boldsymbol{m})$, montre que l'image réciproque du fibré $\mathcal{H}_{\tilde{Q}}^{q}(\mathcal{F})$ est localement plat au dessus de $G \boldsymbol{m}$ et qu'au sens donné plus haut (voir aussi [21]) sa $G$-équivariance est alors "issue de la base" (ce qui signifie que les sections localement constantes sont précisément celles sont localement $G$-invariantes). Le faisceau des germes de ces sections localement constantes sera donc désigné par $\mathcal{H}_{\check{\mathcal{Q}},\left[D^{1,0}=0\right]}^{q}(\mathcal{F})$.

Exploitons cette observation dans le cas plus précis où $M=G / H$ est homogène sous $G$, auquel cas le faisceau $\mathcal{H}_{\tilde{\mathcal{Q}},\left[D^{1,0}=0\right]}^{q}(\mathcal{F})$ est un système local qui définit, comme dans la définition 2.1, $\mathcal{H}_{\mathcal{Q}}^{q}(\mathcal{F})$ comme $\boldsymbol{U}\left(\mathfrak{g}, \mathcal{O}_{M}\right)$-module avec l'action issue de la base au moyen de l'égalité :

$$
\mathcal{H}_{\mathcal{Q}}^{q}(\mathcal{F})=\mathcal{O}_{M} \otimes_{\mathbb{C}} \mathcal{H}_{\mathcal{Q},\left[D^{1,0}=0\right]}^{q}(\mathcal{F})
$$

Désignons alors par $H^{0}$ la composante connexe du sous-groupe de Lie complexe fermé $H \subset G$, et par $\mathfrak{h}$ l'algèbre de $H^{0}$ sur laquelle $H$ agit par la représentation adjointe. Pour fixer les idées, soit $R: H \rightarrow V$ une représentation linéaire complexe holomorphe. Alors ( $c f . \quad[6],[\mathbf{1 9}]$ et $[\mathbf{2 1}]) E \stackrel{\text { def }}{=} G \times{ }^{R} V$ est un fibré vectoriel holomorphe $G$-équivariant; On se placera dans le cas où le $\boldsymbol{U}\left(\mathfrak{g}, \mathcal{O}_{M}\right)$-modules $\mathcal{F}$ est celui des germes de sections holomorphes de $E$. Abusivement on écrira aussi $\mathcal{F}=E$. Par le produit tensoriel de la puissance extérieure de la représentation coadjointe et de $R$, on sait que $H$ agit sur l'espace des cochaînes $C^{q}(\mathfrak{h}, V)$ et que cette $H$-action commute à la différentielle de Koszul :

$$
C^{q}(\mathfrak{h}, V) \stackrel{\delta}{\rightarrow} C^{q+1}(\mathfrak{h}, V) .
$$

La $H$-action sur $H^{q}(\mathfrak{h}, V)$ qui en résulte contient $H^{0}$ dans son ineffectivité i.e. la $H$-action se factorise par une $\Gamma \stackrel{\text { def }}{=} H / H^{0}$-action. Tout ce qui précède permet alors de voir sans trop de difficultés, que le faisceau des sections localement constantes du 
système local

$$
\mathcal{H}_{\mathcal{Q},\left[D^{1,0}=0\right]}^{q}(\mathcal{F})=G \times \times^{\prime \prime} H / H^{0 \prime \prime} H^{q}(\mathfrak{h}, V)
$$

est précisément le $q^{\text {eme }}$ faisceau de cohomologie

$$
\mathcal{H}_{D^{1,0}}^{q} \stackrel{\text { def }}{=} \mathcal{H}^{q}\left(\boldsymbol{C}^{\bullet}(\mathfrak{g}, E)\right)=\mathcal{E} x t_{\boldsymbol{U}\left(\mathfrak{g}, \mathcal{O}_{M}\right)}^{q}\left(\mathcal{O}_{M}, E\right) .
$$

La cohomologie totale $\mathbf{H}^{p+q}(\mathfrak{g}, E)$ est donc approchée par

$$
E_{2}^{p, q}=H^{p}\left(M, \mathcal{H}_{\tilde{\mathcal{Q}},\left[D^{1,0}=0\right]}^{q}\right),
$$

où, dans le deuxième membre, il s'agit de la cohomologie d'un système local au dessus de $M \stackrel{\text { def }}{=} G / H$.

Posons maintenant $\widetilde{M} \stackrel{\text { def }}{=} G / H^{0}$. Via la flèche $G / H^{0} \rightarrow G / H$, c'est le revêtement universel de $M$. Evidemment $\pi_{1}(M)=\Gamma \stackrel{\text { def }}{=} H / H^{0}$, lorsque agissant à gauche par l'action opposée. Une autre suite spectrale, encore due à Grothendieck, permet d'affirmer qu' une graduation de $E_{2}^{p, q}$ est elle-même approchée (avec $p_{1}+p_{2}=p$ ) par

$$
H^{p_{1}}\left(\Gamma ; H^{p_{2}}(\widetilde{M}, \mathbb{C}) \otimes_{\mathbb{C}} H^{q}(\mathfrak{h}, V)\right),
$$

où il faut ici comprendre que $H^{p_{2}}(\widetilde{M}, \mathbb{C}) \otimes_{\mathbb{C}} H^{q}(\mathfrak{h}, V)$ est le $p_{2}^{\text {eme }}$ groupe de cohomologie du système local sur $\widetilde{M}$ obtenu par image réciproque de $\mathcal{H}_{\tilde{Q},\left[D^{1,0}=0\right]}^{q}$ via $\tilde{M} \rightarrow M$. Cette image réciproque est en fait un système local globalement constant, mais la $\Gamma$ équivariance de ce système constant n'est pas triviale; plus précisément la structure de $\Gamma$-module que nous considérons sur $H^{p_{2}}(\widetilde{M}, \mathbb{C}) \otimes_{\mathbb{C}} H^{q}(\mathfrak{h}, V)$ provient simplement de la $\Gamma$-action, évoque plus haut et non nécessairement triviale, sur le deuxième facteur $H^{q}(\mathfrak{h}, V)$.

Si on se rappelle qu'une classe de cohomologie totale dans $\mathbf{H}^{q}(\mathfrak{g}, E)$ définit une $q$-extension :

$$
0 \rightarrow E \rightarrow A_{q} \rightarrow A_{q-1} \rightarrow \cdots \rightarrow A_{1} \rightarrow \mathcal{O}_{M} \rightarrow 0
$$

de $\boldsymbol{U}\left(\mathfrak{g}, \mathcal{O}_{M}\right)$-modules, on peut se poser la question de savoir, par exemple, qu'elles sont les extensions représentées par une classe dans l'image du morphisme latéral :

$$
H^{q}\left(\Gamma, H^{0}(\mathfrak{h}, V)\right) \rightarrow \mathbf{H}^{q}(\mathfrak{g}, E) .
$$

En fait si on considère l'espace d'Eilenberg-Mac Lane $K(\Gamma, 1)$, la représentation de $\Gamma=\pi_{1}(K(\Gamma, 1))$ dans $H^{0}(\mathfrak{h}, V)$ définit un système local $\boldsymbol{\Lambda}$ de fibre type $H^{0}(\mathfrak{h}, V)$ au dessus de $K(\Gamma, 1)$. On sait alors qu'une classe de cohomologie de $H^{q}\left(\Gamma, H^{0}(\mathfrak{h}, V)\right)$ définit une classe d'extensions (au sens de Baer) de systémes locaux sur $K(\Gamma, 1)$ :

$$
0 \rightarrow \boldsymbol{\Lambda} \rightarrow \boldsymbol{L}_{q} \rightarrow \boldsymbol{L}_{q-1} \rightarrow \cdots \rightarrow \boldsymbol{L}_{1} \rightarrow \mathbb{C} \rightarrow 0
$$

Par l'image réciproque de l'application "classifiante" (définie à une homotopie près) $M \stackrel{C}{\rightarrow} K(\Gamma, 1)$, on obtient une extension de systèmes locaux sur $M$. Mais alors les images réciproques $C^{*} \boldsymbol{L}_{k}$ sont des systèmes locaux au dessus de $M$. Si bien qu'avec l'action issue de la base, les fibrés holomorphes sous-jacents $L_{k}^{M} \stackrel{\text { def }}{=} \mathcal{O}_{M} \otimes_{\mathbb{C}} C^{*} \boldsymbol{L}_{k}$ sont 
$\operatorname{des} \boldsymbol{U}\left(\mathfrak{g}, \mathcal{O}_{M}\right)$-modules. Par ailleurs,

$$
\Lambda^{M}=\mathcal{O}_{M} \otimes_{\mathbb{C}} C^{*} \boldsymbol{\Lambda}
$$

est le sous $\boldsymbol{U}\left(\mathfrak{g}, \mathcal{O}_{M}\right)$-module de $E$ engendré par les sections localement $G$-invariantes. C'est-à-dire aussi si l'on veut, le sous-module

$$
\Lambda^{M}=\mathcal{O}_{M} \otimes_{\mathbb{C}} \mathcal{E} x t_{\boldsymbol{U}\left(\mathfrak{g}, \mathcal{O}_{M}\right)}^{0}\left(\mathcal{O}_{M}, E\right) \hookrightarrow E,
$$

avec l' inclusion équivariante qui provient tout simplement de l'inclusion $H$-équivariante

$$
H^{0}(\mathfrak{h}, V) \hookrightarrow V .
$$

Les fibrés holomorphes sur $M$ sous-jacents à ces systèmes locaux constituent une $q$ extension de fibrés holomorphes localement plats qui est donc, pour l'action issue de la base $(c f .[\mathbf{2 1}])$, une $q$-extension de $\boldsymbol{U}\left(\mathfrak{g}, \mathcal{O}_{M}\right)$-modules :

$$
0 \rightarrow \Lambda^{M} \rightarrow L_{q}^{M} \rightarrow L_{q-1}^{M} \rightarrow \cdots \rightarrow L_{1}^{M} \rightarrow \mathcal{O}_{M} \rightarrow 0
$$

Mais l'inclusion $\Lambda^{M} \hookrightarrow E$ de $\boldsymbol{U}\left(\mathfrak{g}, \mathcal{O}_{M}\right)$-module permet par "push out" à partir de la $q$-extension (12) d'en définir une autre :

$$
0 \rightarrow E \rightarrow A_{q} \rightarrow A_{q-1} \rightarrow \cdots \rightarrow A_{1} \rightarrow \mathcal{O}_{M} \rightarrow 0
$$

qui est celle que nous recherchions.

\section{Annexe brève.}

Donnons rapidement des précisions sur l'algèbre multilinéaire en caractéristique zéro. Soit $\mathbf{R}$ un anneau commutatif de caractéristique zéro (dans la pratique il s'agira souvent d'un corps) et M le R-module libre de type fini de rang $n$. Son dual $\mathrm{M}^{*} \stackrel{\text { def }}{=}$ $\operatorname{Hom}_{\mathbf{R}}(\mathbf{M}, \mathbf{R})$ est alors lui aussi libre de type fini et de rang $n$.

Si $p \leqslant r$, on définit le produit intérieur "J" d'une " $r$-forme", i.e. dans $\wedge^{r} \mathbf{M}^{*}$, par un " $p$-vecteur", i.e. dans $\wedge^{p} \mathbf{M}$, comme une " $(r-p)$-forme", et on écrit, si $V^{p} \in \wedge^{p} \mathbf{M}$ et $\omega^{r} \in \wedge^{r} \mathbf{M}^{*}$,

$$
\left.V^{p}\right\rfloor \omega^{r} \in \wedge^{r-p} \mathbf{M}^{*}
$$

Concrètement, si $e_{1}^{1}, \ldots, e_{*}^{r}$ sont dans $\mathbf{M}^{*}$ et $e_{1}, \ldots, e_{p}$ sont dans $\mathbf{M}$ et tels que $e_{*}^{i}\left(e_{j}\right)=\delta_{j}^{i}$ (symbole de Kronecker), alors

$$
\left.\left(e_{1} \wedge \cdots \wedge e_{p}\right)\right\rfloor\left(e_{*}^{1} \wedge \cdots \wedge e_{*}^{r}\right)=e_{*}^{p+1} \wedge \cdots \wedge e_{*}^{r} ;
$$

et avec cette définition on a la formule :

$$
\left.\left.\left.\left(V_{p} \wedge V_{q}\right)\right\rfloor=(-1)^{p q} V_{p}\right\rfloor V_{q}\right\rfloor .
$$

La référence [6] donne une définition ( $c f$. Ann. 15.1.1) du produit intérieur différente de la nôtre, ce qui explique que sa formule (cf. 15.3.1. p. 351) ne soit pas la même que celle donnée ici immédiatement plus haut. Les définitions données ici coincident par contre exactement avec celles données dans [22].

Toujours avec $p \leqslant r$, et en intervertissant les rôles joués par $\mathbf{M}$ et $\mathbf{M}^{*}$. (A vrai dire seule n'intervient ici que la flèche $\mathbf{M} \otimes \mathbf{M}^{*} \rightarrow \mathbf{R}$.) On peut définir le produit intérieur 
d'un " $r$-vecteur" $V_{r}$ par une " $p$-forme" $\omega^{p}$ pour obtenir un $(r-p)$-vecteur. Mais alors pour manifester cette distinction on utilise alors la notation $V^{r}\left\lfloor\omega^{p}\right.$ pour désigner ce produit intérieur pour lequel donc

$$
V^{r}\left\lfloor\left(\omega^{p} \wedge \omega^{q}\right)=\left(V ^ { r } \lfloor \omega ^ { p } ) \left\lfloor\omega^{q} .\right.\right.\right.
$$

Si maintenant $\mathbf{R}$ est un corps de caractéristique zéro, et que $\mathbf{M}$ est un espace vectoriel de dimension $n$, une "forme volume" $\operatorname{vol}_{\mathbf{M}}$, sera un générateur de $\wedge^{n} \mathbf{M}^{*}$. On peut alors démontrer, si $V^{p}$ est un $p$-vecteur et $\alpha \in \mathbf{M}^{*}$, l'identité :

$$
\left.\left(V_{p}\lfloor\alpha)\right\rfloor \operatorname{vol}_{\mathbf{M}}=(-1)^{p+1} \alpha \wedge\left(V_{p}\right\rfloor \operatorname{vol}_{\mathbf{M}}\right) .
$$

\section{Références}

[1] C. Banica and O. Stanasila, Méthodes algébriques dans la théorie des espaces complexes, Collection Varia Mathematica. Gauthier-Villars, Paris, 1977.

[2] H. Cartan and S. Eilenberg, Homological Algebra, Princeton University Press (Fifth Edition 1966), New Jersey, 1956.

[3] S. Chemla, Poincaré duality for $K-A$ Lie superalgebras, Bull. Soc. Math. de France 122 (1994), 371-397.

[4] S. Chemla, A duality property for complex Lie algebroids Math. Z. 232 (1999), 367-388.

[5] N. Chriss and V. Ginzburg, Representation Theory and Complex Geometry, Birkhäuser, Boston MA, Basel, Berlin, 1997.

[6] J. Dieudonné, Éléments d'Analyse, tome 3, Gauthiers-Villars, Paris $2^{\text {eme }}$ édition, 1974.

[7] J. Dixmier, Algèbres Enveloppantes, Gauthiers-Villars, Paris, 1974.

[8] H. Esnault, Characteristic classes of flat bundles, Topology 27 (1988), 323-352.

[9] H. Feld'man, Global dimension of rings of differential operators, Trans. Moscow Mathematical Society 1 (1982), 123-147.

[10] R. Godement, Topologie Algébrique et Théorie des Faisceaux, Hermann, Paris, 1958.

[11] A. Grothendieck, Sur quelques points d'algèbre homologique, Tohoku Journal 9 (1957), 119-221.

[12] P. J. Hilton and U. Stammbach, A Course in Homological Algebra, Graduate Texts in Mathematics, 4. Springer Verlag, Berlin-Heidelberg-New-York, 1970.

[13] F. Hirzebruch, Topological Methods in Algebraic Geometry, Grundlehren der Mathematik 131, Springer-Verlag, Berlin-Heidelberg-New-York, 1966.

[14] J. Huebschmann, Duality for Lie-Rinehart algebras and the modular class, J. Reine Angew. Math. 510 (1999), 103-159.

[15] J. Huebschmann, Differential Batalin-Vilkovisky algebras arising from twilled Lie-Rinehart algebras, Banach Center Publications 51 (2002), 87-102.

[16] F. Lescure, Action sur la cohomologie de Dolbeault, C. R. Acad. Sci. 314 (1992), 923-926. 
[17] F. Lescure, Action non triviale sur la cohomologie de Dolbeault, C. R. Acad. Sci. 316 (1993), 823-825.

[18] F. Lescure, Exemples d'actions induites non résolubles sur la cohomologie de Dolbeault, Topology 35 (1996), 561-581.

[19] F. Lescure, Cohomologie totale et courants de Dolbeault invariants, J. Reine Angew. Math. 475 (1996), 103-136.

[20] F. Lescure, Errata pour l'article Cohomologie totale et courants de Dolbeault invariants, J. Reine Angew. Math. 523 (2000), 235-236.

[21] F. Lescure, Annulation d'une deuxième différentielle, J. Reine Angew. Math. $\mathbf{5 2 7}$ (2000), 37-68.

[22] A. Lichnerowicz, Théorie globale des connexions et des groupes d'holonomie, Edizioni Cremonese, Roma, 1962.

[23] A. Lichnerowicz, Géométrie des groupes de transformations, Dunod, Paris, 1958.

[24] R. S. Palais, A global formulation of the Lie theory of transformations groups, Mem. Amer. Math. Soc. 22 (1957).

[25] G. S. Rinehart, Differential forms for general commutative algebras, Trans. Amer. Math. Soc. 108 (1963), 195-222.

[26] E. Spanier, Algebraic Topology, Series in higher mathematics 9, McGraw-Hill, New York, 1966.

François Lescure

Francois.Lescure@math.univ-lille1.fr, lescurefrancois@yahoo.fr

Université de Lille 1, CNRS-UMR 8524, 59655 Villeneuve d'ASCQ Cedex, France 